ARTICLE

\title{
Loss of microglial SIRP $\alpha$ promotes synaptic pruning in preclinical models of neurodegeneration
}

\author{
Xin Ding (1) 1, Jin Wang ${ }^{1,2,4}$, Miaoxin Huang ${ }^{1,4}$, Zhangpeng Chen ${ }^{1,4}$, Jing Liu' ${ }^{1}$, Qipeng Zhang (1) 1,3,
} Chenyu Zhang ${ }^{1}$, Yang Xiang ${ }^{1}$, Ke Zen (1D) ${ }^{1 凶}$ \& Liang Li (i) ${ }^{1,3 凶}$

Microglia play a key role in regulating synaptic remodeling in the central nervous system. Activation of classical complement pathway promotes microglia-mediated synaptic pruning during development and disease. CD47 protects synapses from excessive pruning during development, implicating microglial SIRP $\alpha$, a CD47 receptor, in synaptic remodeling. However, the role of microglial SIRP $\alpha$ in synaptic pruning in disease remains unclear. Here, using conditional knock-out mice, we show that microglia-specific deletion of SIRP $\alpha$ results in decreased synaptic density. In human tissue, we observe that microglial SIRP $\alpha$ expression declines alongside the progression of Alzheimer's disease. To investigate the role of SIRP $\alpha$ in neurodegeneration, we modulate the expression of microglial SIRP $\alpha$ in mouse models of Alzheimer's disease. Loss of microglial SIRP $\alpha$ results in increased synaptic loss mediated by microglia engulfment and enhanced cognitive impairment. Together, these results suggest that microglial SIRP $\alpha$ regulates synaptic pruning in neurodegeneration.

\footnotetext{
${ }^{1}$ Nanjing Drum Tower Hospital Center of Molecular Diagnostic and Therapy, Chinese Academy of Medical Sciences Research Unit of Extracellular RNA, State Key Laboratory of Pharmaceutical Biotechnology, Nanjing University, Nanjing, China. ${ }^{2}$ Department of Endocrinology, Drum Tower Hospital Affiliated to Nanjing University Medical School, Nanjing, China. ${ }^{3}$ Institute for Brain Sciences, Nanjing University, Nanjing, China. ${ }^{4}$ These authors contributed equally: Xin Ding, Jin Wang, Miaoxin Huang, Zhangpeng Chen. ${ }^{凶}$ email: kzen@nju.edu.cn; liangli@nju.edu.cn
} 
S ubstantial evidence has demonstrated that microglia play a pivotal role in synapse remodeling ${ }^{1-3}$. Dysfunction of microglia-mediated synaptic pruning during neurodevelopment results in abnormal social behavior in adulthood ${ }^{4,5}$. Furthermore, such malfunction is also implicated in several disease-associated neurological symptoms ${ }^{6-8}$. Removing the redundant synapses correctly is important for the normal development and functional homeostasis of the healthy brain, which requires collaboration of both positive and negative signals modulating synaptic elimination ${ }^{9}$.

Previous studies mainly focused on the signals that positively regulate microglia-mediated synaptic pruning ${ }^{10,11}$. The innate immune molecules, $\mathrm{Clq}$ and $\mathrm{C} 3$, have been demonstrated to promote microglial engulfment of synaptic structures via activating $\mathrm{C} 3$ receptor $(\mathrm{CR} 3)^{12,13}$. Another microglial chemokine receptor, $\mathrm{Cx} 3 \mathrm{cr} 1$, is also required for microglial pruning during neurodevelopment and whose deficiency undermines neural refinement and cause social behavior defects ${ }^{2,14}$. These signals not only control synapse remodeling in early neurodevelopment, but also play crucial roles in several neurological diseases ${ }^{13,15,16}$. Alzheimer's Disease $(\mathrm{AD})$ is an aging related neurodegenerative disorder, in which synapse loss is the major correlate of cognitive impairment ${ }^{17}$. It has been demonstrated that $\mathrm{Clq}$ level is significantly enhanced in neuronal synapses in the early stage of $\mathrm{AD}$, resulting in the consequent synaptic loss, while blocking the signal significantly alleviates neurodegeneration in $\mathrm{AD}$ mice model ${ }^{18}$. The abnormality of complement system may also cause neurological symptoms in several other diseases such as virus infection and schizophrenia, accompanied by aberrant microgliamediated synapse loss ${ }^{16,19,20}$. These results suggest that appropriate microglia-mediated synapse elimination is important for maintaining normal brain function.

Despite much research conducted on the signals that positively modulate synaptic clearance, the negative regulatory pathways involved in microglia-mediated synaptic pruning are less known. Until recently, Lehrman et al. ${ }^{9}$ have discovered that neuronal CD47, which can bind to SIRPa and produce "do not eat me" signal, protects synapses from excessive elimination. CD47 null mice have less synapses with increased microglial pruning in dorsal lateral geniculate nucleus (dLGN), indicating the potential protective role of SIRP $\alpha-\mathrm{CD} 47$ axis in synapses refinement during neurodevelopment ${ }^{9}$.

SIRPa (also known as SHPS-1, p84, and BIT) is a transmembrane protein that binds to its ligand, $\mathrm{CD} 47$, activating the protein tyrosine phosphatases SHP-1 or SHP-2 through its cytoplasmic region ${ }^{21}$. We have demonstrated that SIRP $\alpha$ expresses in microglia and regulates immune response as well as phagocytic activity 22 . Despite the work regarding to neuronal CD47, it is still unclear whether microglial SIRPa is actually involved in the negative regulation of synaptic removal. Moreover, the potential role of SIRPa-CD47 axis in synaptopathological diseases such as $\mathrm{AD}$ remains to be investigated.

In the present study, we have demonstrated that synaptic density is significantly reduced after microglial SIRPa is ablated in newborn mice, along with excessive synapse pruning by microglia. SIRPa deficiency in microglia undermines its ability to recognize CD47 signal and increases phagocytosis towards synaptic elements. Furthermore, we have provided evidence that microglial SIRPa level declines in the progression of AD. Inhibition of microglial SIRPa signal remarkably exacerbates synaptopathology and cognitive impairment by promoting aberrant synaptic elimination in $\mathrm{AD}$ mice model. Taken together, our results suggest that microglial SIRPa negatively regulates synaptic removal, which plays a critical role under both physiological and pathological conditions.

\section{Result}

Microglial SIRPa specific ablation decreased synaptic density in brain. First, we assessed SIRPa expression level according to the cell type and developmental stages (P5, P30, and P60). Immunostaining assay revealed that SIRP $\alpha$ primarily expressed in neuron and microglia while there was little expression in astrocyte (Supplementary Fig. 1a). Neuronal SIRPa expression showed a time dependent increase during development stage (Supplementary Fig. 1b, d). Microglial SIRPa level was high at P5, which declined at P30 and maintained this low level at P60 (Supplementary Fig. 1c, e-g). There was no obvious spatial heterogeneity of microglial SIRPa expression in P5 or P30 mice brain (Supplementary Fig. $1 \mathrm{~h}-\mathrm{j}$ ). In order to evaluate the contribution of microglial SIRPa in synapse pruning during neurodevelopment, we generated an inducible microglial SIRPa knockout mouse line-Cx3cr1 ${ }^{\text {CreERT2 }}: S I R P a^{\mathrm{fl} / \mathrm{fl}}$ mice (Supplementary Fig. 2a). Mice $\left(\mathrm{Cx} 3 \mathrm{cr} 1^{\mathrm{CreERT} 2}: \mathrm{SIRPa}^{\mathrm{fl} / \mathrm{fl}}\right.$ mice) received three consecutive injections of tamoxifen (TAM) once a day from P1 to P3 (Supplementary Fig. 2b). PCR and flow cytometric analysis showed TAM-induced DNA recombination resulted in stable deletion of SIRPa in microglia (CD45 ${ }^{\text {int }}$ CD11b $b^{\text {high }}$ cells) (Supplementary Fig. 2c, d).

We applied $\mathrm{Cx} 3 \mathrm{cr} 1^{\mathrm{CreERT} 2}: \mathrm{SIRPa}{ }^{\mathrm{fl} / \mathrm{fl}}$ mice treated with corn oil as control. SIRPa ${ }^{\mathrm{fl} / \mathrm{fl}}$ mice receiving TAM were included as another control to rule out potential side-effects caused by tamoxifen. Mice $\left(\mathrm{Cx} 3 \mathrm{cr} 1^{\mathrm{CreERT}}: \mathrm{SIRPa}^{\mathrm{fl} / \mathrm{fl}}\right)$ treated with TAM were referred as microglial SIRPa specific knockout mice (SIRPacKO mice; Fig. 1a).

NeuN staining suggests that the number of neurons and the size of the cell body are not changed in SIRPa-cKO mice (Supplementary Fig. 3a-d, g). Neuronal SIRPa expression was not affected either (Supplementary Fig. 3e, f). Additionally, microglial density and branches were not altered in SIRPa-cKO mice (Supplementary Fig. 4). The expression of SIRP $\beta 1$, a closely related family member of SIRPa, was not changed in SIRPadeficient microglia (Supplementary Fig. 5a-c). To assess the synaptic morphology in SIRPa-cKO mice, we conducted Homer1 and Vglut1 double staining in primary visual cortex (V1) and hippocampus (CA1) at different time points (P5, P15, and P30). Synaptic density was not altered at P5 or P15 in V1 cortex of SIRPa-cKO mice, while it significantly decreased at P30 compared to control (Fig. 1b, d-f and Supplementary Fig. 6a, c, e). The initial synaptic number at P5 or P15 did not alter significantly, indicating that synaptic reduction at P30 is less likely due to early neuron growth defect. Meanwhile, synaptic number in hippocampus of SIRPa-cKO mice showed remarkable decrease at P15 and P30, which occurs earlier than that in primary visual cortex (Fig. 1c, g-i and Supplementary Fig. 6b, d, f). Furthermore, we demonstrated that microglial SIRPa deficiency decreased the frequency, but not the amplitude, of miniature excitatory postsynaptic currents (mEPSCs) in CA1 neuron of P30 mice (Fig. 1j-n), which is coincident with the reduction of synaptic contacts in hippocampus.

Homer1 and Vglut2 double staining revealed that synaptic density was significantly decreased in dLGN of SIRPa-cKO mice at P15 and P30 while these signals remained equivalent at P5 (Supplementary Fig. 7a-d). We further analyzed eye-specific retinogeniculate segregation pattern, which was not affected at P5 but showed significant decrease in overlap between ipsilateral and contralateral signals at $\mathrm{P} 10$ and $\mathrm{P} 30$, respectively (Supplementary Fig. 7e-h). In addition, SIRPa-cKO and control mice displayed similar innervation of the dLGN in P5 (Supplementary Fig. 7i, j). These data together suggest that microglial SIRPa deficiency decreases synaptic density without affecting neuron growth at early stage $(<\mathrm{P} 5)$. 


\section{a}

SIRPa ${ }^{n / 1 / 1}(+T A M P 1-P 3)$

CX3cr1 $1^{\text {CreERT2}}:$ SIRPa ${ }^{\text {fl/f }}(-$ TAM)

Cx3cr1 ${ }^{\text {CreERT2:SIRPa }}{ }^{\text {fl/f }}(+$ TAM P1-P3)
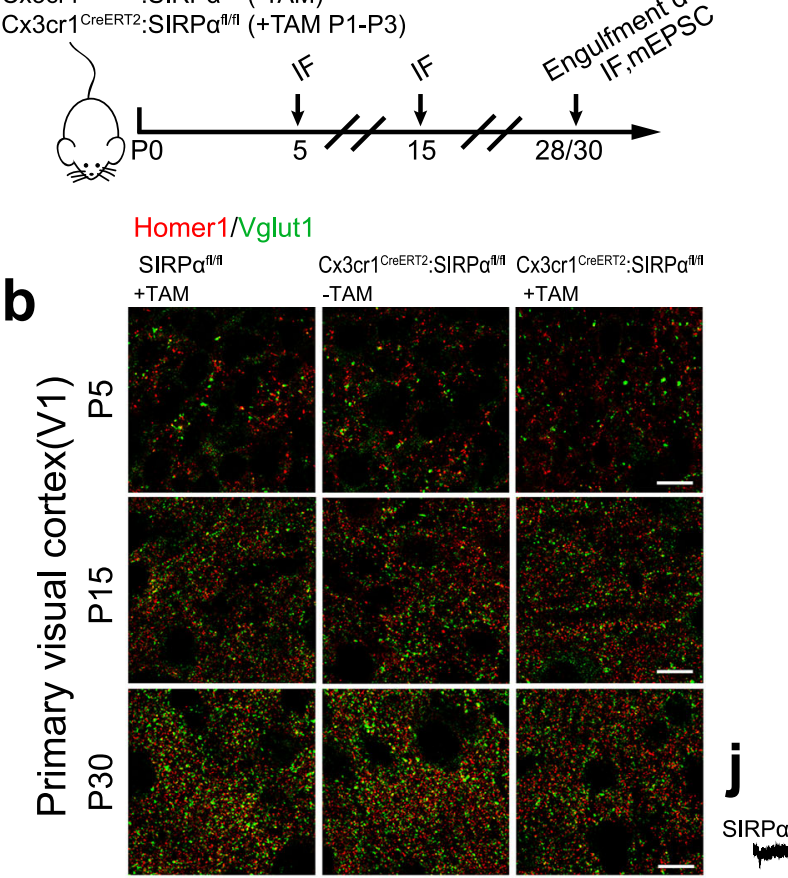

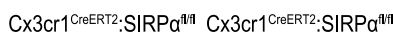

C

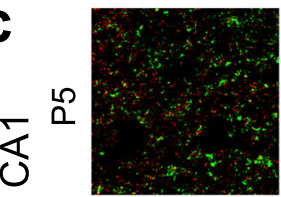

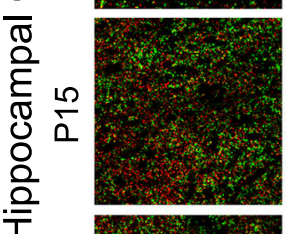

品

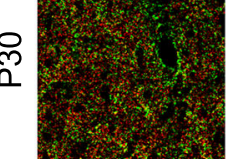

O

SIRPa +TAM

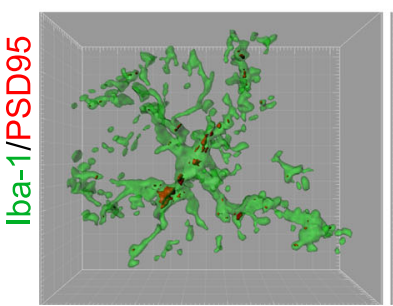

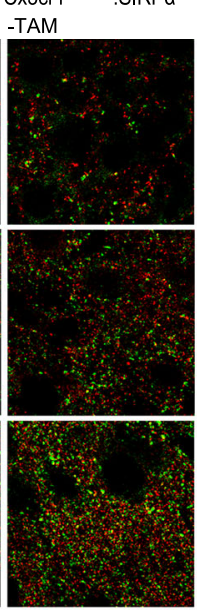
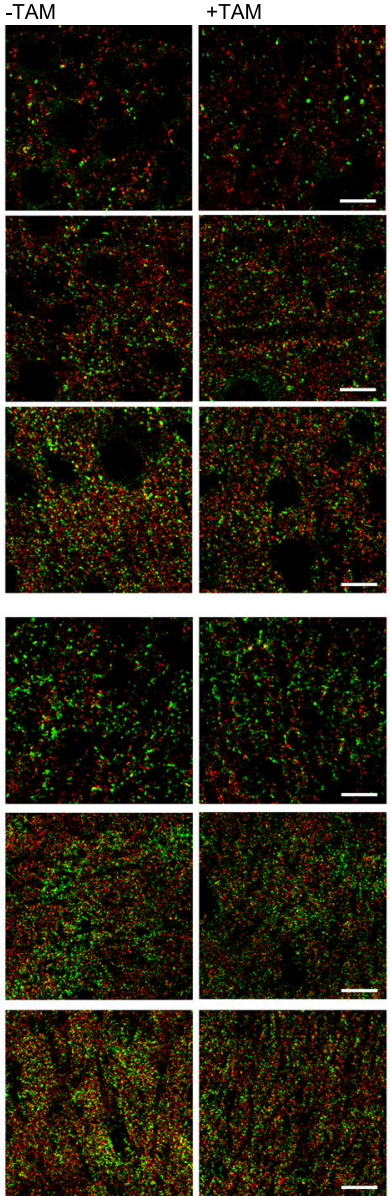

CX3cr1 ${ }^{\text {CreERT2:SIRPa }}{ }^{\text {fl/fl }}$ - TAM

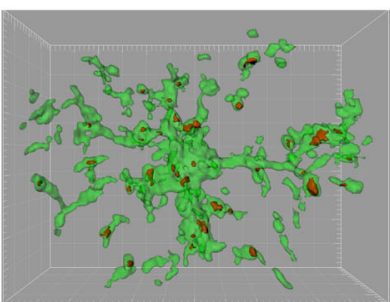

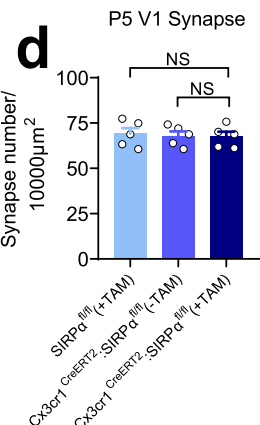
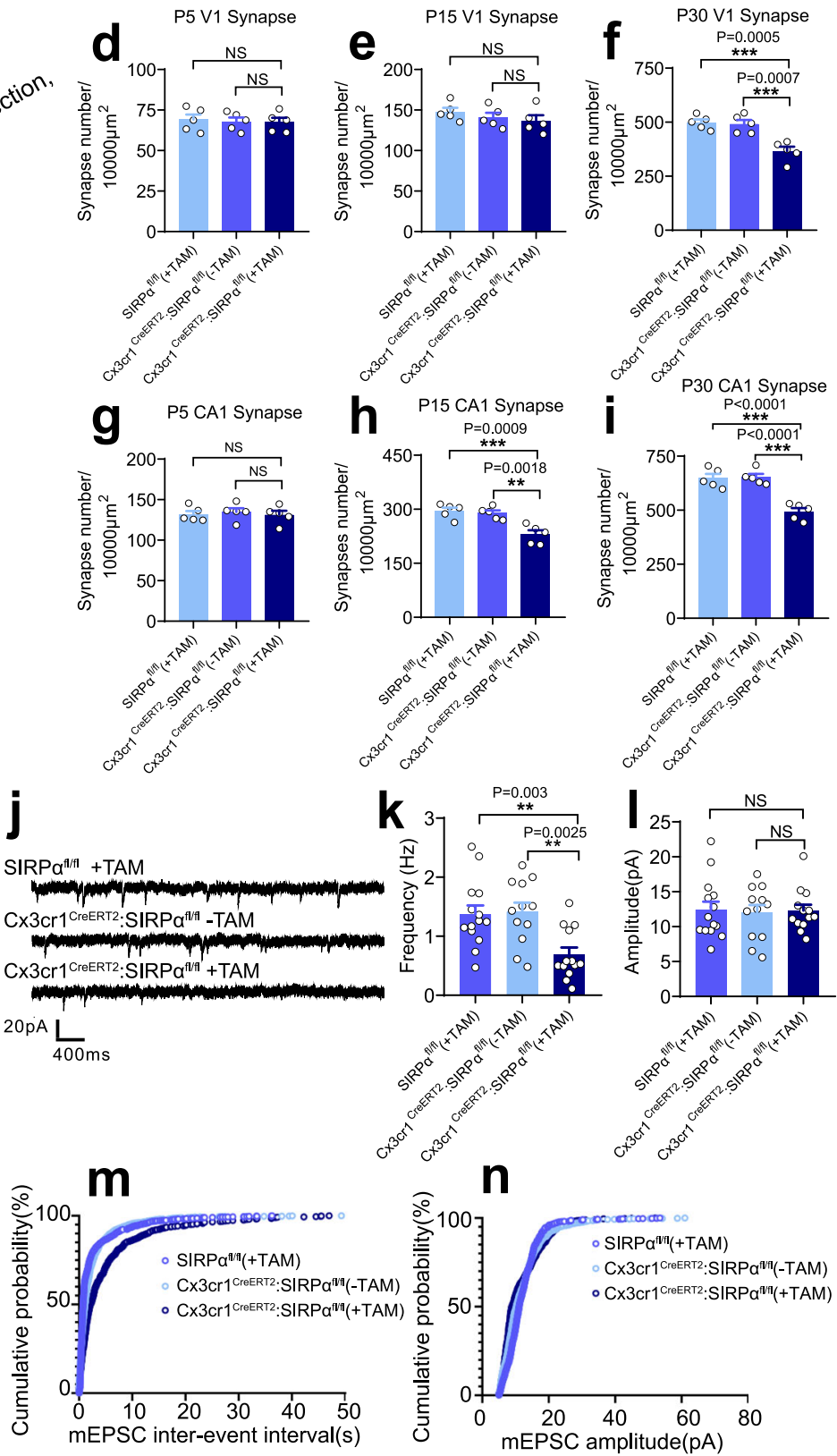

Cx3cr1 $1^{\text {CreERT2:SIRPa }}{ }^{\mathrm{n} / \mathrm{fl}}$ + TAM
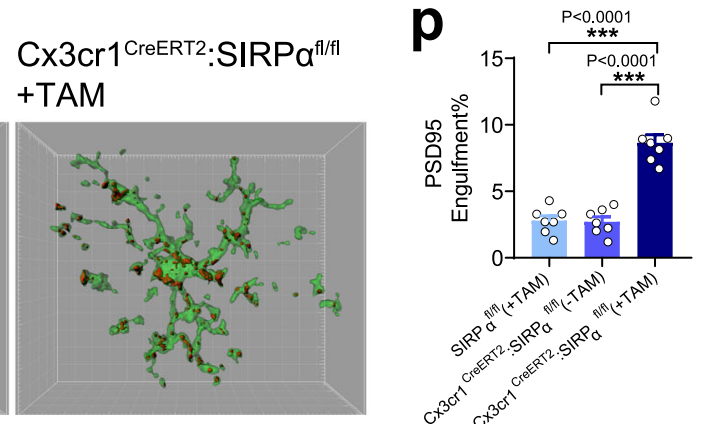

Increased engulfment of synaptic structure in SIRPa deficient microglia. To investigate the role of microglia in the synaptic density reduction we observed in SIRPa-cKO mice, we measured microglial engulfment of synaptic elements in cortex (V1) at P28. A negative control that only use secondary antibody demonstrated the specificity of synaptic marker labeling (Supplementary Fig. 8). There was larger volume ratio of $\mathrm{PSD} 5^{+}$or Vglut $^{+}$ puncta in Iba-1 positive microglia of SIRPa-cKO mice compared to control (Fig. 1o, p and Supplementary Fig. 6g, h). We also assessed microglial phagocytosis towards synaptic elements in vitro using neuron-microglia co-culture system (Fig. 2a). Iba-1/ MAP2 staining revealed high purify $(>90 \%)$ of primary microglia/ neurons before coculturing (Supplementary Fig. 9a, b). Both PSD95 and synaptophysin (SPH) staining showed that the overall 
Fig. 1 Synaptic density in microglial SIRP $\alpha$ deficient mice is lower on account of increased microglial engulfment of synaptic structures. a Schematics of the experimental procedures. IF immunofluorescence, mEPSC miniature excitatory postsynaptic current, TAM tamoxifen, P postnatal. Neonatal mice were injected $50 \mu \mathrm{g}$ tamoxifen at P1, P2, and P3. b, c Representative confocal images depict synaptic staining for presynaptic marker Vglut1 (green) and

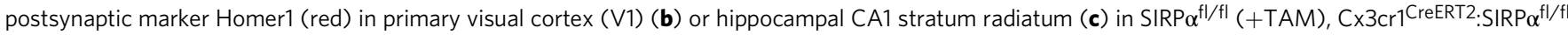

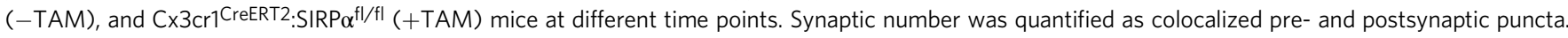
Scale bar, $10 \mu \mathrm{m}$. d-i Histograms depict synaptic density in V1 (d-f) or hippocampal CA1 (g-i) in those mice. $n=5$ mice/group; average of 10-12 fields from each mouse. One-way ANOVA analysis with Dunnett's multiple comparisons test. $\mathbf{j}$ Example traces of mEPSCs (miniature excitatory postsynaptic currents)

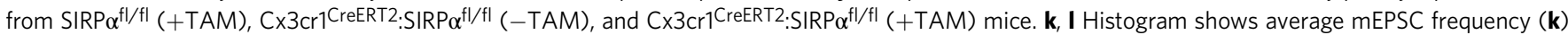

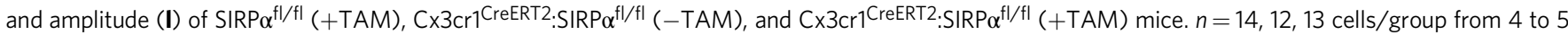
mice. One-way ANOVA analysis with Dunnett's multiple comparisons test. $\mathbf{m}, \mathbf{n}$ Cumulative probability distributions of mEPSC inter-event intervals ( $\mathbf{m}$ ) and

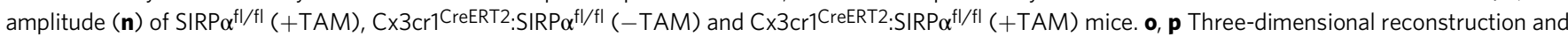
surface rendering demonstrate larger volumes of PSD95 puncta inside lba-1 positive microglia in V1 from P28 Cx3crl ${ }^{\mathrm{CreERT2}}: \mathrm{SIRP} \alpha^{\mathrm{fl} / \mathrm{fl}}(+\mathrm{TAM})$ mice. $n=7$ mice/group, average of 8-9 microglia from each mouse, one-way ANOVA analysis with Dunnett's multiple comparisons test. Grid line increments $=5 \mu$ m. Data are mean \pm s.e.m. ${ }^{\star \star} P<0.01,{ }^{\star \star \star} P<0.001$, NS not significant. Detailed statistical information was listed in Supplementary Statistical Data. Source data are provided as a Source Data file.
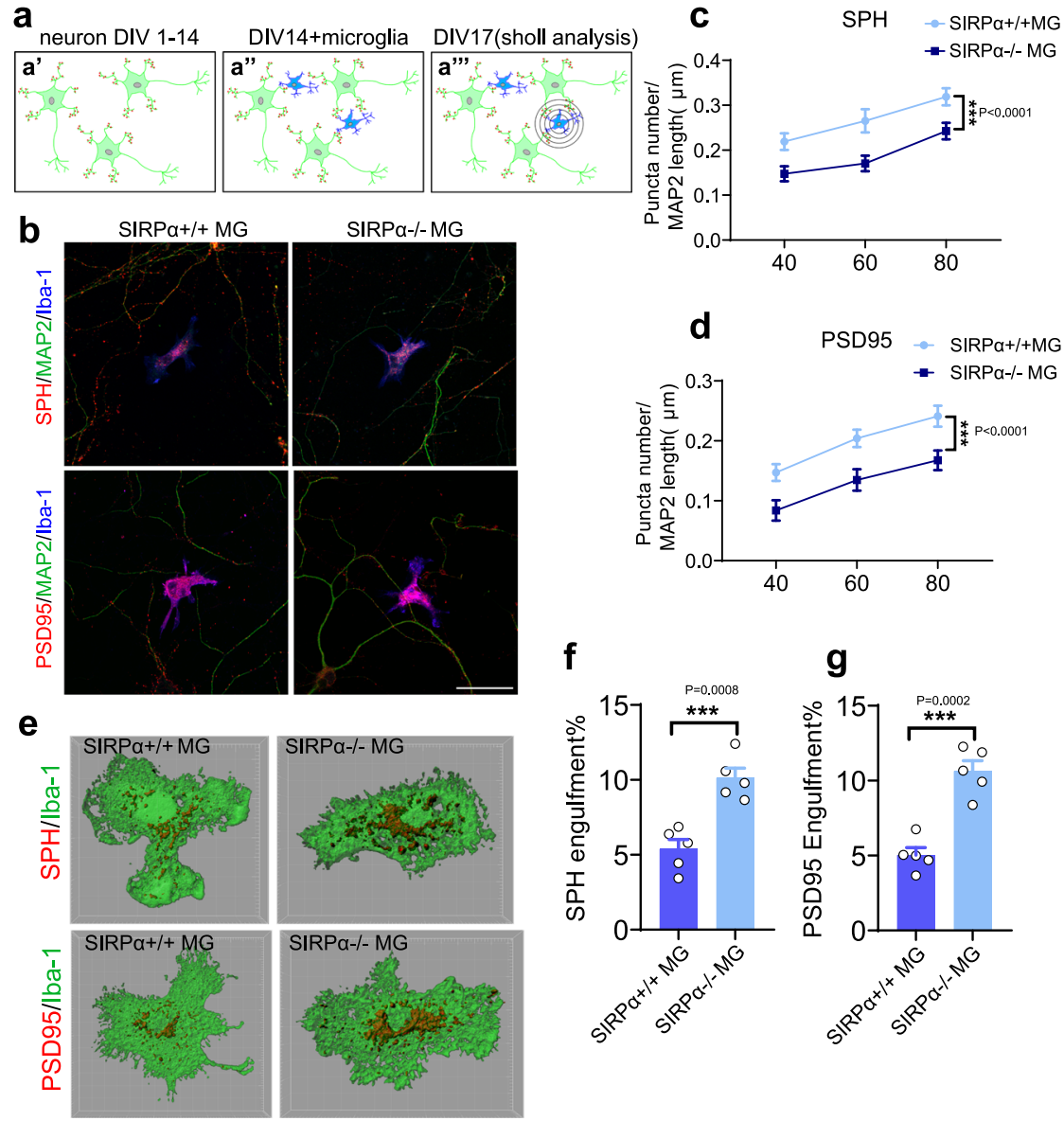

f

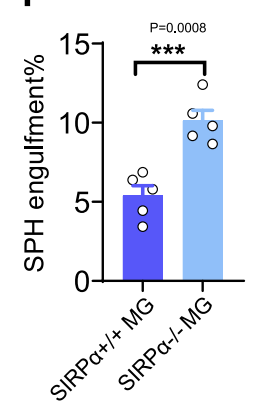

g

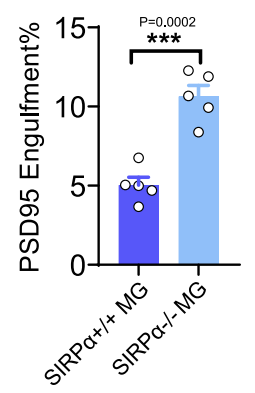

Fig. 2 Primary cultured SIRP $\boldsymbol{\alpha}$ deficient microglia engulf more synaptic elements. a Diagrams show neuron-microglia co-cultures (a' and a") and Sholl analyses ( $\left(\mathrm{a}^{\prime \prime}\right)$ that quantifies synaptic puncta around microglia. Green cells in diagrams represent neurons (MAP2 $2^{+}$, red puncta represent synaptic marker (SPH/PSD95), and blue cells represent microglia (Iba- $\left.1^{+}\right)$. b-d Confocal images (b) show the presence of synaptic puncta $\left(\mathrm{SPH}^{+} / \mathrm{PSD} 5^{+}\right)$around SIRP $\alpha^{+/+}$ or SIRPa $\alpha^{-/-}$microglia $\left(\mathrm{Iba}^{+}\right)$. Scale bar, $50 \mu \mathrm{m}$. Statistical analysis for Syanptophysin (c) or PSD95 density (d) is obtained by two-way ANOVA; $n=5$ independent experiments. e-g $3 \mathrm{D}$ reconstruction and surface rendering demonstrate that SIRP $\alpha^{-/-}$microglia engulf more synaptic elements. Grid line increments $=5 \mu \mathrm{m}$. Histograms depict the statistics of synaptic engulfment. Two-tailed unpaired $t$-test, $n=5$ independent experiments, average of 3-4 microglia from each experiment. SPH synaptophysin, MG microglia. Data are mean \pm s.e.m. ${ }^{\star \star \star} P<0.001$. Detailed statistical information was listed in Supplementary Statistical Data. Source data are provided as a Source Data file.

synaptic density (in area without microglia) was not altered after adding a few microglia with different genotype in the primary neuron culture (Supplementary Fig. 9c, d). However, those $\mathrm{PSD}^{+}{ }^{+}$or $\mathrm{SPH}^{+}$synaptic density adjacent to $\mathrm{SIRPa}^{-1-}$ microglia (within $80 \mu \mathrm{m}$ ) were significantly reduced compared to control (Fig. 2b-d). In the co-culture system, microglial morphology (area and circularity) was not significantly altered in SIRPa deficient groups (Supplementary Fig. 9e, f). Most of these microglia $(>90 \%)$ contained PSD95 or SPH positive structures, which were equivalent in both groups (Supplementary Fig. 9g). We further quantified fluorescent signal of synaptic markers (PSD95 or SPH) inside microglia using 3D reconstruction and 
a

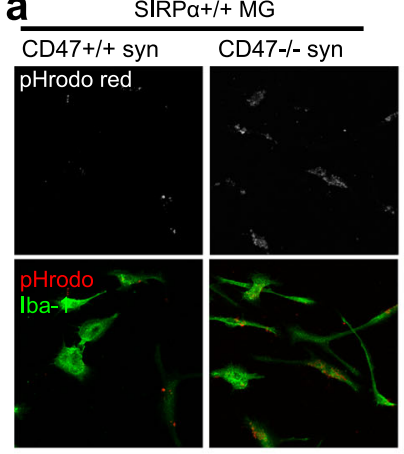

C

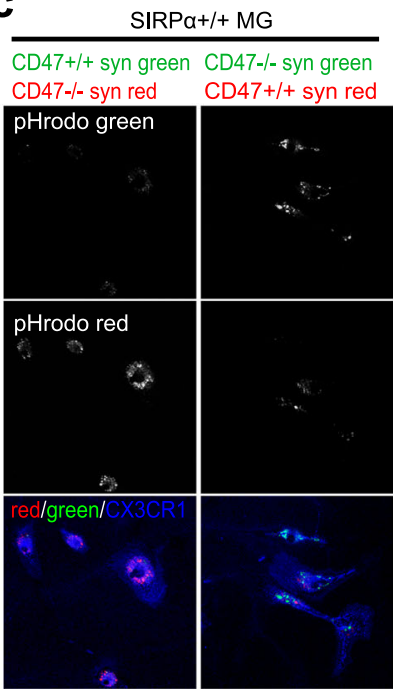

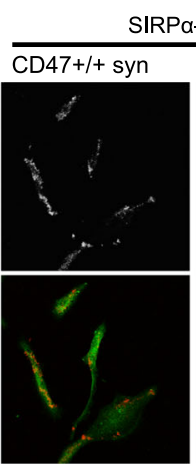

SIRPa-I-MG

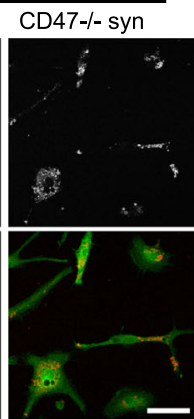

d

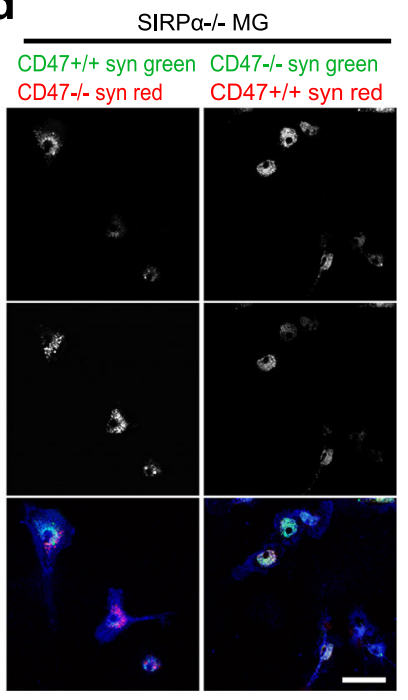

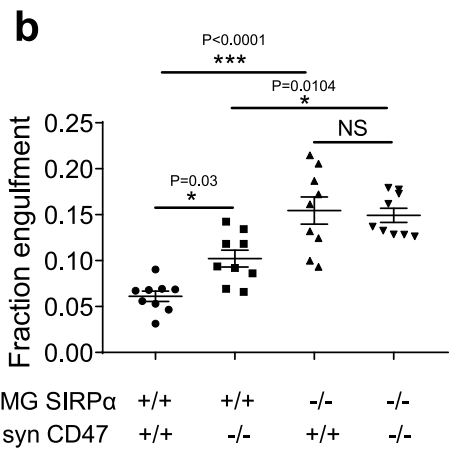

e

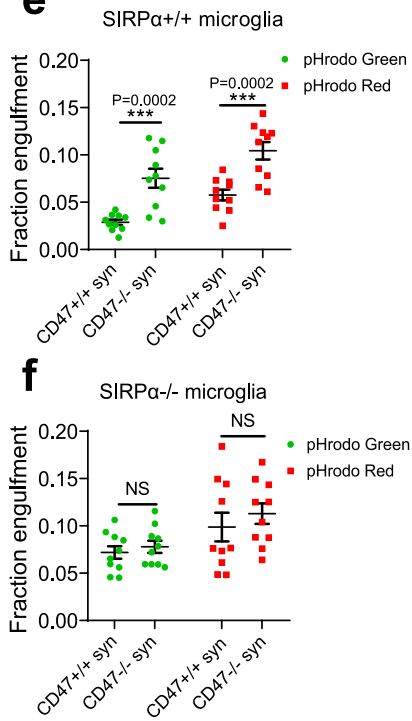

Fig. 3 SIRP $\alpha$ deficient microglia show increased phagocytic activity. a Representative images show that primary cultured microglia (SIRP $\alpha+/+$, $\mathrm{SIRP} \alpha^{-/-}$) phagocytosed pHrodo red-conjugated synaptosomes (CD47+/+, CD47-/-). Scale bar, $50 \mu \mathrm{m}$. b Graph depicts the fraction of synaptosomes engulfed by microglia of different genotypes. $n=9$ wells; average of 5-6 fields from each well. One-way ANOVA analyses followed by Tukey's multiple comparison test. c, d Representative images show that CX3CR1 labeled SIRP $\alpha^{+/+}$(c) or SIRP $\alpha^{-/-}$(d) microglia engulfed a mixture of synaptosomes $\left(\mathrm{CD} 47^{+/+}: \mathrm{CD} 47^{-/-}=1: 1\right)$ conjugated with pHrodo-red or pHrodo-green respectively. Scale bar, $50 \mu \mathrm{m}$. e, f Graph depicts the fraction of synaptosomes engulfed by SIRP $\alpha^{+/+}(\mathbf{e})$ or SIRP $\alpha^{-/-}(\mathbf{f})$ microglia for each combination of pHrodo color. $n=10$ wells; average of 5-6 fields from each well. Data were analyzed by two-way ANOVA analysis via Sidak's multiple comparisons test. Data are mean \pm s.e.m. ${ }^{\star} P<0.05,{ }^{\star \star \star} P<0.001, \mathrm{NS}$ not significant. Detailed statistical information was listed in Supplementary Statistical Data. Source data are provided as a Source Data file.

surface rendering method, and the results revealed more synaptic structures in SIRPa deficient cells (Fig. 2e-g). Additionally, we have demonstrated that microglia engulfed more pHrodo Redconjugated synaptosomes when SIRPa-CD47 signal is interrupted (Fig. 3a, b). Meanwhile, SIRPa deficient microglia displayed increased phagocytic activity compared to control (Fig. 3a, b).

To further testify whether SIRPa deficiency undermines the ability of microglia to identify CD47 signal, mixture of CD $47^{+/+}$ or $\mathrm{CD} 47^{-1-}$ synaptosomes labeled with different pHrodo dye were cultured with microglia. Afterwards, we quantified microglial engulfment and demonstrated that $\mathrm{SIRPa}^{+/+}$microglia preferred to engulf $\mathrm{CD} 47^{-1-}$ synaptosomes (Fig. $3 \mathrm{c}$, e). On the contrary, SIRPa deficient microglia were not able to discriminate the CD47 signal and exhibited indistinguishable phagocytic capacity towards both types of synaptosomes (Fig. 3d, f). These data suggest that SIRPa deficiency in microglia lead to increased phagocytic ability as well as compromised recognition of CD47 signal, which may further result in the excessive elimination of synaptic structures.
Similar results have been achieved in CD47-KO mice. Both morphological and electrophysiological data suggested that synaptic density was reduced in cortex as well as in hippocampus of these mice (Fig. 4a-n; Supplementary Fig. 10). In the meantime, microglia-mediated synaptic clearance during development is remarkably enhanced (Fig. 4o, p). Together, these data indicate that SIRPa-CD47 signal axis protects synapses from excessive elimination mediated by microglia during early neurodevelopment.

Synaptic CD47 is regulated by neural activity. Immunostaining results demonstrated that there were both $\mathrm{PSD}^{+} 5^{+} / \mathrm{CD} 47^{+}$and $\mathrm{PSD}^{+} / \mathrm{CD}^{-} 7^{-}$structures in the neurites of primary neurons (Fig. 5a). Similar observation was obtained when we labeled neurons with CD47 and Synaptophysin (Fig. 5b). We also conducted Vglut1/CD47 or PSD95/CD47 double labeling in brain sections and observed both $\mathrm{CD} 47^{+}$and $\mathrm{CD} 47^{-}$synaptic elements in vivo (Fig. 5c). These data indicate that CD47 may differentially expressed in neuronal synapses. We further isolated synaptosomes 
a
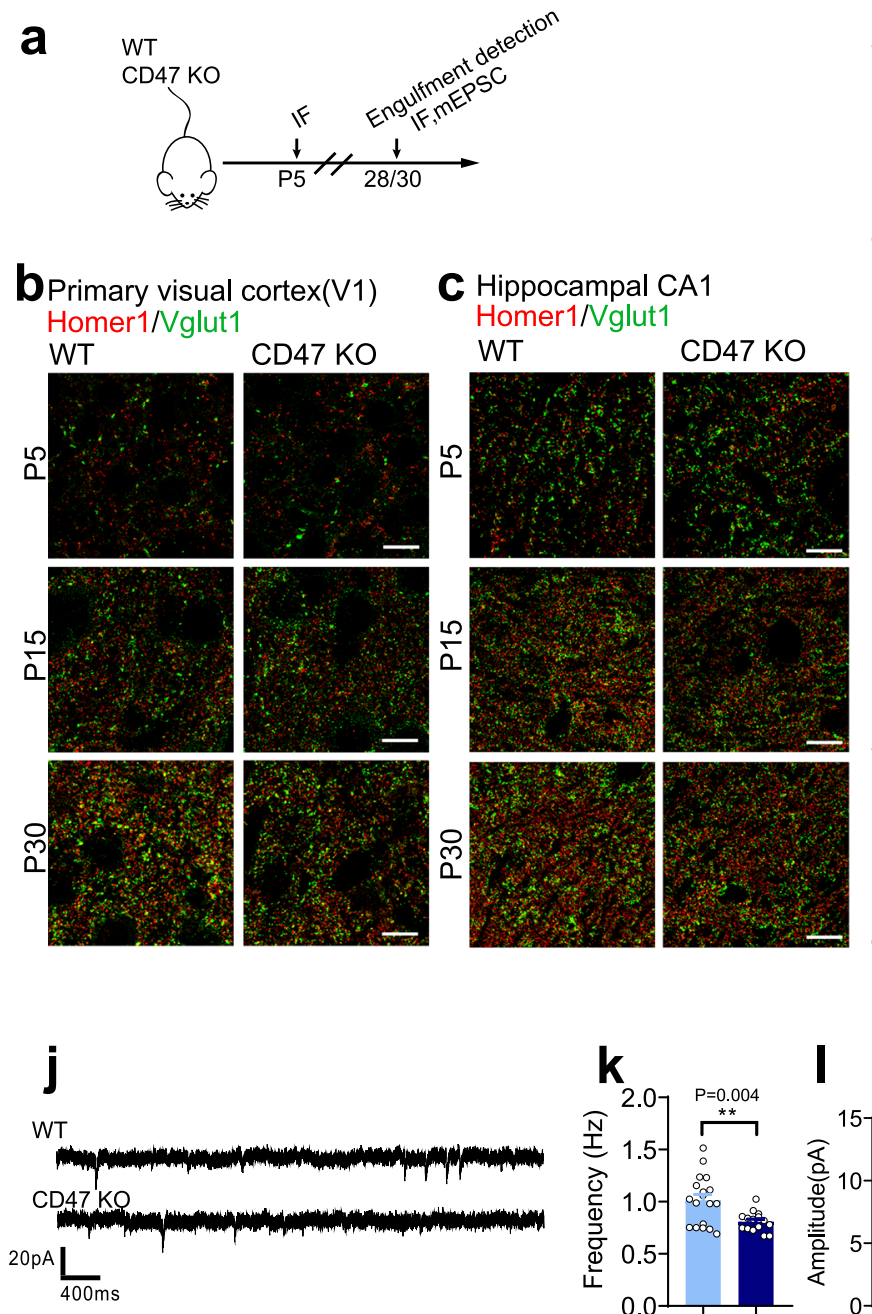

n

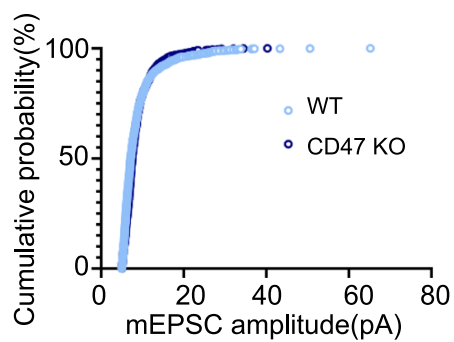

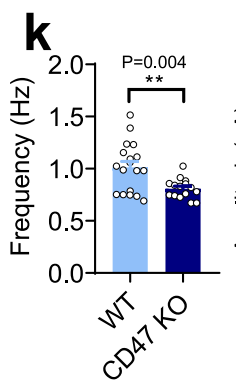

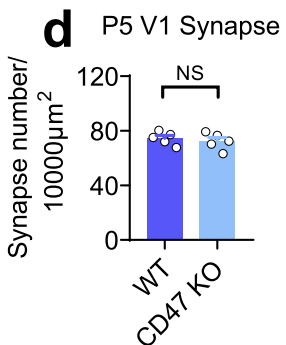

e P15 V1 Synapse
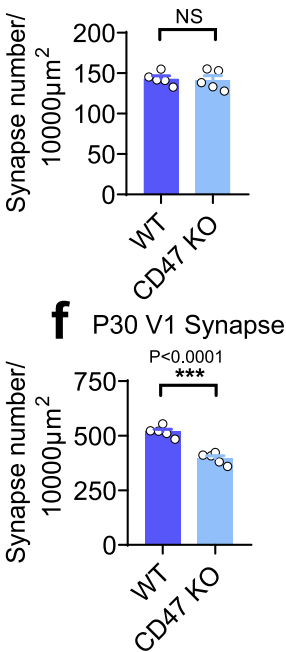

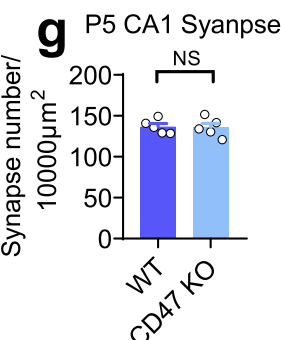

h P15 CA1 Synapse
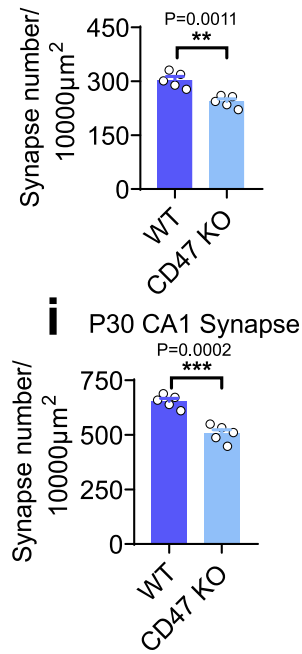

m

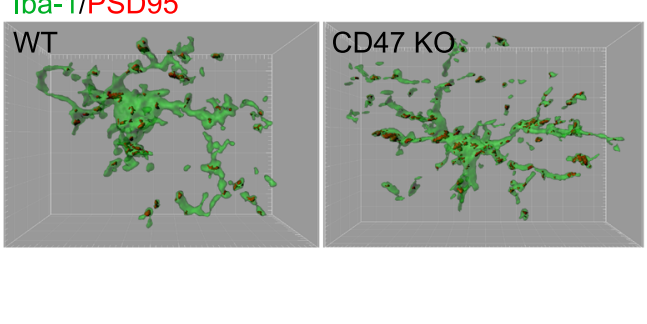

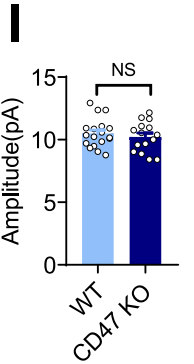

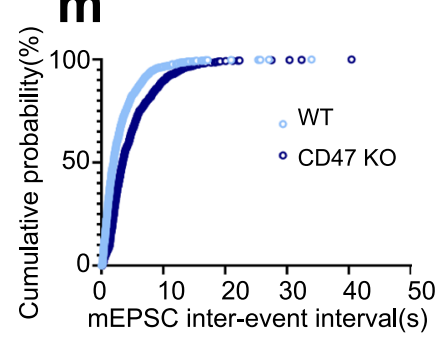

p

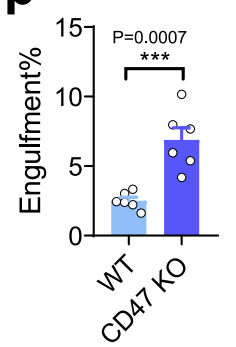

Fig. 4 Synaptic density in CD47-KO mice is lower on account of enhanced microglial engulfment of synaptic structures. a Schematics of the experimental procedures. IF immunofluorescence, mEPSC miniature excitatory postsynaptic current. b, c Representative confocal images depict synaptic staining for presynaptic marker Vglut1 (green) and postsynaptic marker Homer1 (red) in primary visual cortex (V1) (b) and hippocampal CA1 stratum radiatum (c) in WT and CD47-KO mice at different time points. Synaptic number was quantified as colocalized pre- and postsynaptic puncta. Scale bars, $10 \mu \mathrm{m}$. $\mathbf{d}$-i Histograms depict the relative level of synaptic density in $\mathrm{V} 1(\mathbf{d}-\mathbf{f})$ or hippocampal CA1 ( $\mathbf{g}-\mathbf{i}$ ) in those mice. $n=5$ mice/group; average of $10-12$ fields from each mouse, two-tailed unpaired $t$ test. $\mathbf{j}$ Example traces of mEPSCs from WT and CD47-KO mice. $\mathbf{k}$, I Histogram shows average mEPSC frequency $(\mathbf{k})$ and amplitude (I) of WT and CD47-KO mice. $n=18,15$ cells from 6 to 8 mice, two-tailed unpaired $t$ test. $\mathbf{m}, \mathbf{n}$ Cumulative probability distributions of mEPSC inter-event intervals $(\mathbf{m})$ and amplitude $(\mathbf{n})$ of WT and CD47-KO mice. $\mathbf{0}, \mathbf{p} 3 \mathrm{D}$ reconstruction and surface rendering demonstrate larger volumes of PSD95 puncta inside Iba-1 positive microglia in V1 from P28 CD47-KO mice versus WT mice. $n=6$ mice/group, average of 8-9 microglia from each mouse, two-tailed unpaired $t$ test. Grid line increments $=5 \mu \mathrm{m}$. Data are mean \pm s.e.m. ${ }^{\star \star} P<0.01,{ }^{\star \star \star} P<0.001$, NS not significant. Detailed statistical information was listed in Supplementary Statistical Data. Source data are provided as a Source Data file. 

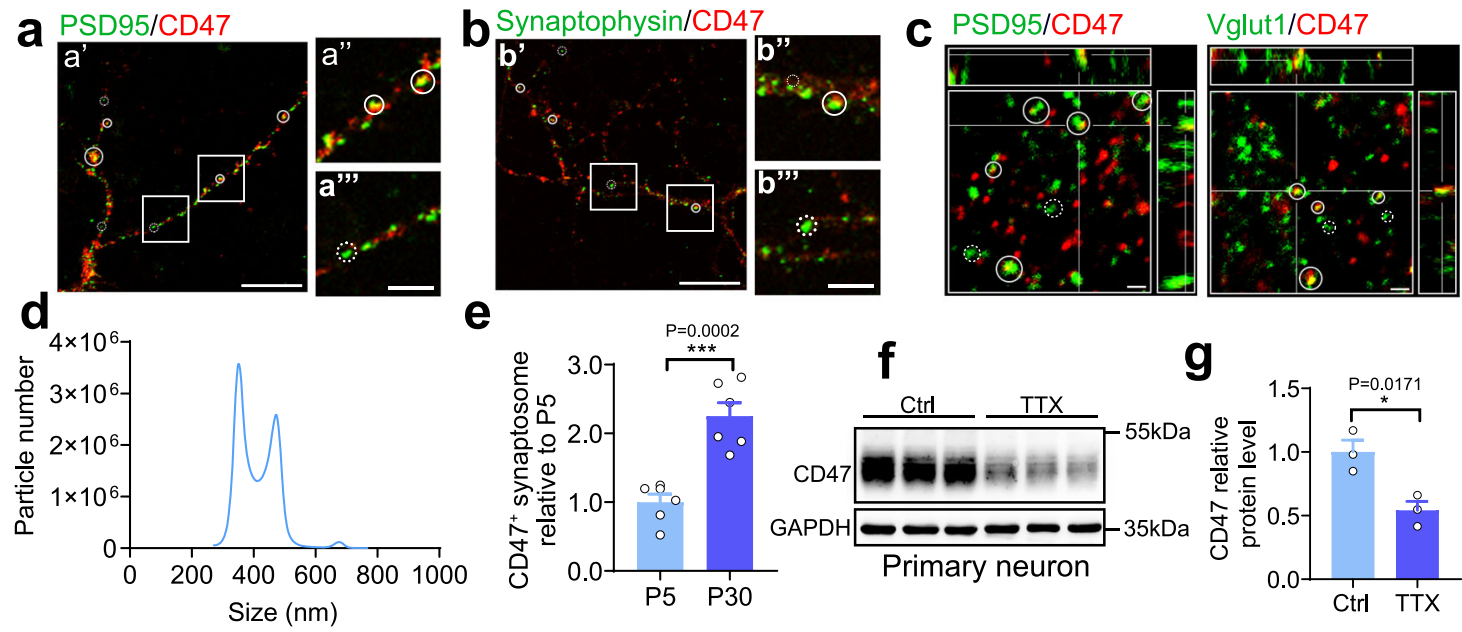

\section{e}

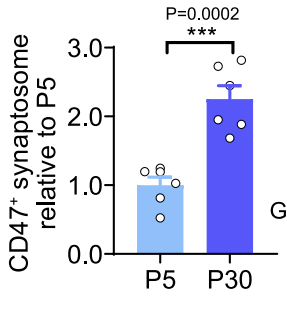

g
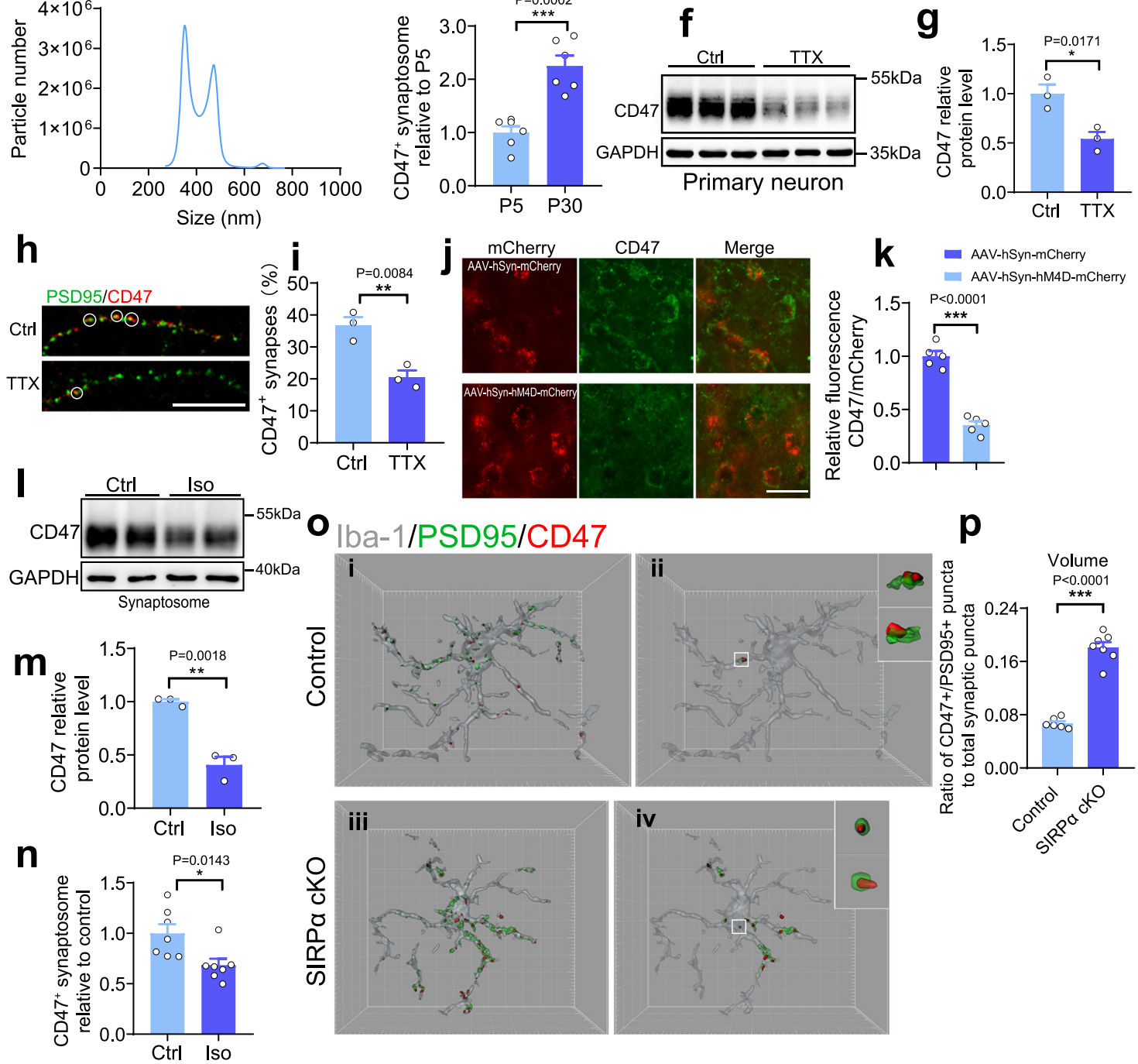

from mice brain and measured CD47 signal by Nanosight. The number of CD47 positive synaptosomes in $\mathrm{P} 30$ mice is significantly higher than that of P5 mice (Fig. 5d, e), suggesting an agedependent increase of $\mathrm{CD} 47$ positive synapses in mice brain. As microglia incline to engulf less active inputs during neural refinement, we hypothesized that low level of neural activity may decrease CD47 signal, which facilitates microglia-mediated synaptic elimination. To assess impact of neural activity on CD47 expression, channel-blocker tetrodotoxin (TTX) was applied and it significantly decreased level of CD47 expression as well as CD47 positive synapses in primary neuron (Fig. $5 \mathrm{f}-\mathrm{i}$ ). We also overexpressed hM4D (Gi) with mCherry in cortical neurons in adult mice (Supplementary Fig. 11). After inhibiting the neuron activity by CNO (clozapine-N-oxide) administration, we measured the protein level of $\mathrm{CD} 47$ in those neurons and found remarkable decrease of CD47 expression compared to control (Fig. 5j, k). Synaptosomes isolated from general anesthetic (isoflurane treated) mice brain showed reduced CD47 expression compared to control
(Fig. 5l, m). Nanosight analysis suggested that number of CD47 positive synaptosomes in anesthetic mice is downregulated as well (Fig. 5n). Moreover, phagocytic assay revealed that microglia preferred to engulf those synaptosomes derived from anesthetic mice brain, indicating CD47 downregulation facilitates microgliamediated synaptic clearance (Supplementary Fig. 12a, b).

We further compared amount of $\mathrm{CD} 47^{+} / \mathrm{PSD} 5^{+}$puncta in microglia from SIRPa-cKO and control mice. In control microglia, only a few of $\mathrm{PSD}^{+} 5^{+}$puncta were $\mathrm{CD} 47$ positive, indicating the preferential engulfment of $\mathrm{CD} 47$ negative synapses. In SIRPa deficient microglia, ratio of $\mathrm{CD} 47^{+} / \mathrm{PSD} 95^{+}$puncta to total PSD95 ${ }^{+}$puncta were significantly increased, suggesting that the selective engulfment of $\mathrm{CD}^{-} 7^{-}$synapses was abolished in microglia after losing SIRPa (Fig. 5o, p). Furthermore, we examined ratio of $\mathrm{CD} 47^{+} / \mathrm{PSD} 5^{+}$synaptic structure in microglia from mice under anesthesia condition. Isoflurane treatment increased microglial engulfment of $\mathrm{PSD}^{+} 5^{+}$synaptic structures (Supplementary Fig. 12c, d). Most of those PSD95 ${ }^{+}$elements 
Fig. 5 CD47 is expressed in synapses and regulated by neural activity. a, b Confocal images show CD47 co-expression with presynaptic marker

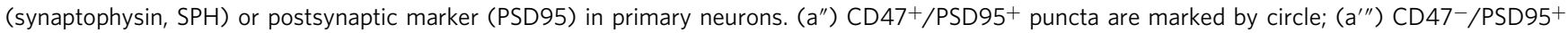
puncta are marked by dashed circle; $\left(b^{\prime}\right) \mathrm{CD} 47^{+} / \mathrm{SPH} 95^{+}$puncta are marked by circle; $\left(b^{\prime \prime}\right) \mathrm{CD} 47^{+} / \mathrm{SPH} 95^{+}$puncta are marked by dashed circle; Scale bars, $\left(a^{\prime}, b^{\prime}\right) 20 \mu \mathrm{m} ;\left(a^{\prime \prime \prime}, b^{\prime \prime \prime}\right) 5 \mu \mathrm{m}$. This experiment was repeated independently for three times. c Orthogonal views of CD47/PSD95 (postsynaptic marker) or CD47/Vglut1 (presynaptic marker) double staining in brain section in P28 mice, CD47+ or CD47- synaptic elements were marked by circleor dashed circle. Scale bar, $1 \mu \mathrm{m}$. This experiment was repeated independently for three times. $\mathbf{d}$ Nanosight shows size range of synaptosomes passed through membrane filters (pore size $0.45 \mu \mathrm{m}$ ). e Synaptosomes conjugated with CD47-FITC antibody are quantified by Nanosight and the relative level of CD47+ synaptosomes in adult mice is significantly increased compared to that in newborn mice. $n=6$ mice/group, two-tailed unpaired $t$ test. $\mathbf{f}$, $\mathbf{g}$ Western blot analysis of primary neurons shows that neuronal CD47 expression was downregulated after TTX treatment $(1 \mu \mathrm{M}$ for $48 \mathrm{~h}) . n=3$ independent experiments, two-tailed unpaired $t$ test. h, i Representative images and statistical analysis show the percentage of CD47+ synaptic structures $(C D 47+$ PSD95 puncta/total PSD95 puncta) was reduced in TTX (tetrodotoxin) treated neurons. Scale bar, $20 \mu \mathrm{m} . n=3$ independent experiments, average 6-7 fields from each assay; two-tailed unpaired $t$ test. $\mathbf{j}$, $\mathbf{k}$ Representative images and statistical analysis demonstrate that CD47 level decreased in neuron expressing the inhibitory DREADD-hM4Di in response to CNO stimulation. AAV-hSyn-hM4D(Gi)-mCherry or control virus ( $\left.100 \mathrm{~nL}, 1.25 \times 10^{13} \mathrm{GC} / \mathrm{mL}\right)$ was injected into mouse primary visual cortex, 4 weeks later mice were subjected to Clozapine- $N$-oxide (CNO) administration for 3 consecutive days. Scale bar, $20 \mu \mathrm{m} ; n=5$ mice/group, average of 8-10 fields from each mouse, two-tailed unpaired $t$ test. I, $\mathbf{m}$ Western blot analysis of synaptosomes isolated from isoflurane (iso) treated mice also shows reduction of CD47 compared to control mice, $n=3$ mice/group, two-tailed unpaired $t$ test. $\mathbf{n}$ Nanosight detection reveals that relative level of $\mathrm{CD}_{4} 7^{+}$synaptosomes isolated from isoflurane (iso) treated mice is significantly reduced. $n=7$ mice/group, unpaired $t$ test. 0 Left panels show $\mathrm{CD}^{4} 7^{+}$or PSD95 ${ }^{+}$signal inside microglia from SIRP $\alpha$-cKO and control mice (i and iii); right panels (ii and iv) show CD47 and PSD95 colocalized puncta inside cells. Insets are enlarged images with orthogonal plane of typical CD47 ${ }^{+} /$PSD $5^{+}$puncta. Grid line increments $=5 \mu \mathrm{m}$. $\mathbf{p}$ Histogram shows volume ratio of $\mathrm{CD} 47^{+} / \mathrm{PSD}^{+} 5^{+}$puncta to total synaptic structures, $n=6,7$ mice/group, average of $8-9$ cell from each mouse, two-tailed unpaired $t$ test. Data are mean \pm s.e.m. ${ }^{\star} P<0.05,{ }^{\star \star} P<0.01,{ }^{\star \star \star} P<0.001$, NS not significant. Detailed statistical information was listed in Supplementary Statistical Data. Source data are provided as a Source Data file.

were $\mathrm{CD} 47$ negative, ratio of $\mathrm{CD} 47^{+} / \mathrm{PSD} 5^{+}$puncta to total synaptic structures showed a little but significant declination compared to mice under normal condition (Supplementary Fig. 12e). Together, these data indicate that CD47 expression may decrease in less active synapses which are prone to be eliminated by microglia.

Microglial SIRPa is downregulated in AD pathology. We further investigated the role of microglial SIRPa in AD, in which loss of synapses is a central neuroanatomical hallmark. The protein level of SIRPa was remarkably decreased in the cortex of $\mathrm{AD}$ patients (Fig. 6a, b). It was also downregulated in AD mice brain (6 months and 12 months old) compared to same-aged control (Supplementary Fig. 13a, b). In the meantime, SIRP $\beta 1$ expression is elevated in both human $\mathrm{AD}$ patients and $\mathrm{AD}$ mice brain (Supplementary Fig. 5d-h).

To specifically assess the alteration of SIRPa in microglia during $\mathrm{AD}$, we conducted flow cytometric analysis and showed that microglial SIRPa did not change significantly in WT mice at different time points ( 2,5 , and 8 months). On the contrary, SIRPa started to decline in microglia of $\mathrm{AD}$ mice at 5 months age (before plaque formation) and continued to decrease at 8 months old (after plaque formation; Fig. 6c, d). Immunostaining assay confirmed the declination of microglial SIRPa in 5- and 8months-old AD mouse brain, while microglial SIRPa levels were similar between $\mathrm{AD}$ and $\mathrm{WT}$ mice at 2 months age (Fig. 6e, $\mathrm{f}$ and Supplementary Fig. $13 \mathrm{c}-\mathrm{f}$ ). When we challenged primary microglia with soluble $A \beta_{42}$ oligomer $(A \beta O)$, the protein level of SIRPa significantly decreased (Fig. 6g, h). Flow cytometric analysis also revealed marked reduction of microglial SIRPa in mice brain followed with intracerebroventricular (ICV) injections of $A \beta O$ (Fig. 6i, j). These data suggest that $A \beta$ oligomer generated in the early stage of AD inhibits SIRPa expression in microglia.

SIRPa deficiency enhanced cognitive impairment and synaptic loss without modifying $\mathbf{A} \boldsymbol{\beta}$ plaque depositions. To exclude the potential impact of neuronal SIRPa as well as the developmental change caused by early microglial SIRPa deletion, we have applied $\mathrm{AD}^{\mathrm{APP}}$ swe, PSEN1dE9/SIRPa-cKO mice model (by tamoxifen induction, microglial SIRPa was specifically ablated at
2 months age) to investigate the role of microglial SIRPa deficiency on AD pathogenesis in adulthood (Fig. 7a). Open field analysis showed similar locomotor activity among all those mice groups (control; SIRPa-cKO; $\mathrm{AD} ; \mathrm{AD} / \mathrm{SIRPa}-\mathrm{cKO}$ ) at 5 months age (Fig. 7b). Although we observed decreased locomotor activity in mice with $\mathrm{AD}$ background at 8 months age, there were no significant differences between $\mathrm{AD}$ and $\mathrm{AD} / \mathrm{SIRPa}-\mathrm{cKO}$ mice (Fig. 7b). In addition, all groups of mice showed similar escape latencies in the visible platform test (Fig. 7c), which implies comparable vision among these mice. Hidden platform test and spatial probe trial results suggested that microglial SIRPa ablation accelerated cognitive impairment in $\mathrm{AD}$ mice at 5 months age, even preceding the plaque formation (Fig. $7 \mathrm{~d}, \mathrm{e}$ ). Although such effect persisted in 8-months-old mice (Fig. 7d, e), we did not find any significant variations in amyloid deposition between $\mathrm{AD}$ and AD/SIRPa-cKO mice at this stage (Fig. 7f, g). ELISA analysis also demonstrated that both soluble and insoluble $\mathrm{A} \beta_{42}$ were not significantly changed between these mice at 5 - or 8 -months age (Fig. 7h, i). Iba-1 and 6E10 double staining revealed that microglial recruitment around plaques between $\mathrm{AD}$ and $\mathrm{AD} /$ SIRPa-cKO mice were indistinguishable (Fig. 7j, k). These data suggest that microglial SIRPa deficiency has little impact on amyloid pathology in AD mice.

PSD95/Synapsin I labeling showed that microglial SIRPa deficiency remarkably enhanced synaptic loss in cortex and hippocampus of $\mathrm{AD}$ mice at 5- and 8-months age (Fig. 7l, $\mathrm{m}$ and Supplementary Fig. 14). Additionally, Golgi staining demonstrated consistent results that dendritic spine density was less in $\mathrm{AD} / \mathrm{SIRP} \alpha-\mathrm{cKO}$ mice (Fig. 7n, o). Notably, ablation of microglial SIRPa at 2-months-age did not alter synaptic number in these mice with non-AD background at 5 months or 8 months age (Fig. 7l-o and Supplementary Fig. 14). These data suggest that microglial SIRPa deficiency in adulthood accelerated synapse loss and cognitive declination in $\mathrm{AD}$ mice.

Loss of microglial SIRPa increased A $\beta$ o-induced synapse loss by promoting microglia-mediated synaptic elimination. We have provided evidence that A $\beta$ o downregulated microglial SIRP $\alpha$ expression. To further specify its role in synaptopathology during $\mathrm{AD}$ progression, we applied another AD model using SIRPa-cKO mice followed with ICV injection of A $\beta$ o (Fig. 8a). Specific gene 


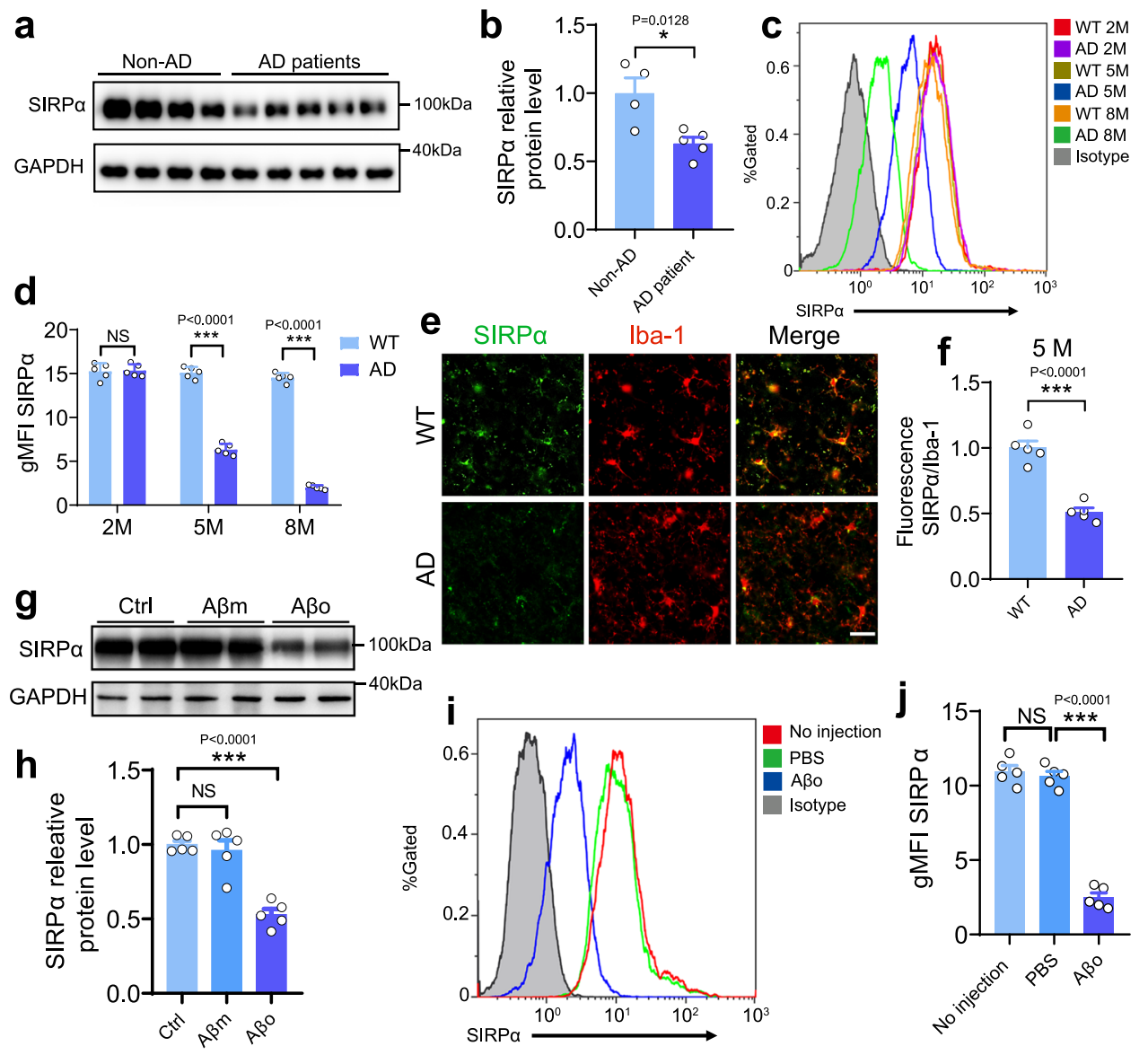

Fig. 6 Microglial SIRPo is downregulated in AD pathology. a, b Western blot and statistical analysis show SIRP $\alpha$ expression decreased remarkably in the cortex of $A D$ patients. Non- $A D=4, A D$ patients $=5$. Two-tailed unpaired $t$ test. Detailed sample information is listed in the Table 1 in Methods section. c, d Flow cytometry analysis displays SIRP $\alpha$ expression of acute isolated microglia from 2-months, 5-months, and 8-months old WT and AD mice. Histograms represent quantification of geometric mean fluorescent intensity (gMFI) of SIRP $\alpha . n=5$ mice/group, average of three tests from each mouse, two-way ANOVA via Sidak's multiple comparisons test. e, $\mathbf{f}$ Immunostaining and quantification of SIRP $\alpha$ with microglial marker (Iba-1) in cortex of WT and AD mice ( 5 months old). Scale bar, $25 \mu \mathrm{m}, n=5$ mice/group; average of 6-8 fields from each mouse, two-tailed unpaired $t$ test. $\mathbf{g}$, $\mathbf{h}$ Western blot and statistical analysis of SIRP $\alpha$ protein level in primary cultured microglia after $A \beta_{42}$ monomer $(A \beta m)$ or $A \beta_{42}$ oligomer (A $\beta$ o) treatment. Cells were treated with $0.2 \mu \mathrm{M} \mathrm{A} \beta$ for $24 \mathrm{~h}$ before protein analysis. $n=5$ experiments, one-way ANOVA, Dunnett's multiple comparisons test. $\mathbf{i}$, $\mathbf{j}$ Flow cytometry analysis shows microglial SIRP $\alpha$ decreased 3 days after intracerebroventricular (ICV) injection of A $\beta$ o $(2 \mu \mathrm{g} / \mathrm{mouse})$. Histogram shows the gMFI quantification of microglial SIRP $\alpha . n=5$ mice/group (WT male, 3 months age), average of three tests from each mouse. One-way ANOVA analysis with Dunnett's multiple comparisons test. Data are mean \pm s.e.m. ${ }^{\star} P<0.05,{ }^{\star \star \star} P<0.001$, NS not significant. Detailed statistical information was listed in Supplementary Statistical Data. Source data are provided as a Source Data file.

deletion is induced by TAM injection in 2-months-old mice to exclude potential impact of microglial $\operatorname{SIRPa}$ deficiency during early neurodevelopment (Fig. 8a). After A $\beta$ o injection (3-monthsold mice), synaptic number significantly reduced in SIRPa-cKO mice compared to that in control (Fig. 8b, c), which is consistent to results we achieved in AD/SIRPa-cKO mice. Similarly, deletion of microglial SIRPa at 2-months-age had little impact on synapses of those mice without $A \beta o$ stimulation (Fig. 8b, c).

We further examined microglial engulfment towards synaptic elements and demonstrated that there was significantly larger volume of PSD95+ structures in microglia of SIRPa-cKO mice after $\mathrm{A} \beta \mathrm{o}$ challenge (Fig. 8d, e). Besides, flow cytometric analysis detected more microglia with PSD95 positive elements in SIRPacKO mice (Fig. 8f, g). These data suggest that phagocytic activity of microglia towards synaptic structures is increased in SIRPa$\mathrm{cKO}$ mice upon $\mathrm{A} \beta$ stimulation, leading to enhanced synaptic loss during $A D$ pathology. In vitro assay revealed that both $A \beta m$ and $A \beta O$ stimulation increased phagocytosis of synaptosomes in primary microglia (Supplementary Fig. 15a, b), which may due to microglia activation. However, $\mathrm{A} \beta \mathrm{O}$ treatment decreased microglial SIRPa expression (Fig. 6g, h), which was associated with greatly enhanced phagocytosis compared to $A \beta m$-treated group (Supplementary Fig. 15a, b). This result suggests that downregulation of SIRP $\alpha$ after $A \beta$ o treatment further increases phagocytosis of synaptosomes in microglia. In addition, overexpressing SIRPa via lentivirus infection remarkably reduced microglial engulfment towards synaptosomes after A $\beta$ o treatment (Supplementary Fig. 15c, d).

To further elucidate the role of SIRPa-CD47 signal axis in AD pathology, we examined CD47 protein level in primary neuron subjected to $A \beta$ stimulation and found that $A \beta o$ treatment significantly decreased CD47 expression (Supplementary Fig. 16a, b). Synaptosome CD47 level was also declined in AD mouse brain at 8 -months age (Supplementary Fig. 16c, d). Those AD mice $(8 \mathrm{M})$ derived synaptosomes were easier to be engulfed by microglia (Supplementary Fig. 16e, f). In addition, we performed ICV injection of $A \beta O$ in CD47-KO mice and found those mice were more vulnerable upon $A \beta o$ stimulation, displaying greater loss of synapses and enhanced microglial engulfment compared to control (Supplementary Fig. $16 \mathrm{~g}-\mathrm{k}$ ). Together, these data 
Control:Cx3cr1 1reeRr2:SIRPa nift (-TAM)

SIRPa CKO:CX3cr1 ${ }^{\text {CreERT2:SIRPa }} \alpha^{\text {UIII }}$ (+TAM 2M)

AD: APPswe, PSEN1dE9

a

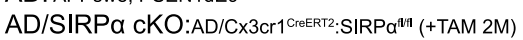

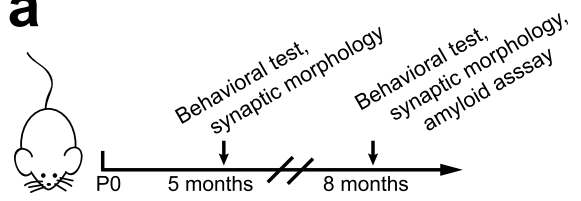

d Hidden platform (5M)
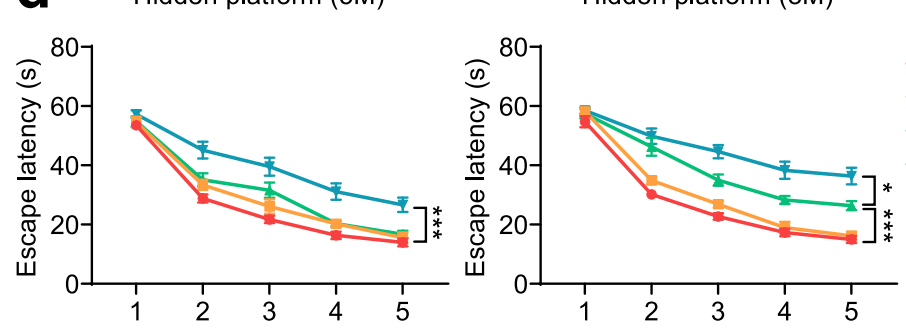

b Open field (5M)

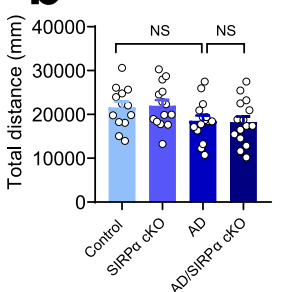

Hidden platform $(8 \mathrm{M})$

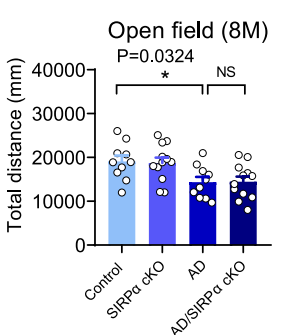

C Visible platform (5M) Visible platform (8M)
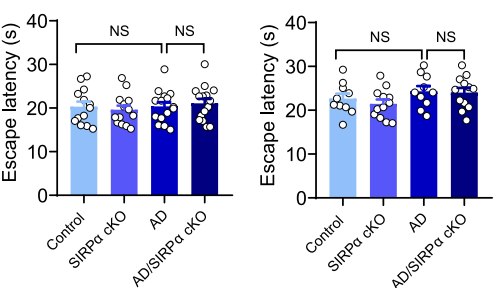

e

Probe trial $(8 \mathrm{M})$

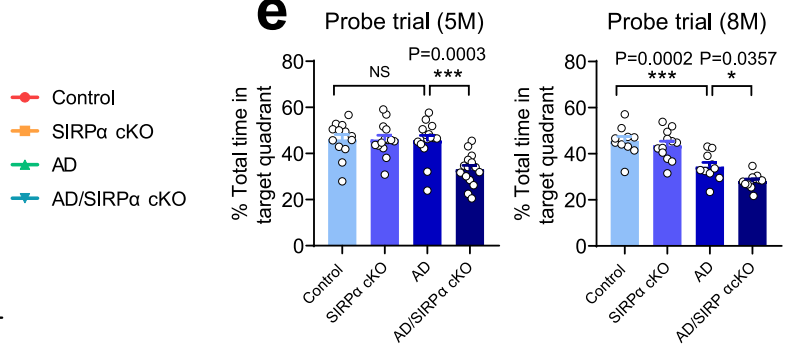

$\mathbf{f}_{6 \mathrm{E} 10 / \mathrm{DAPI}}$
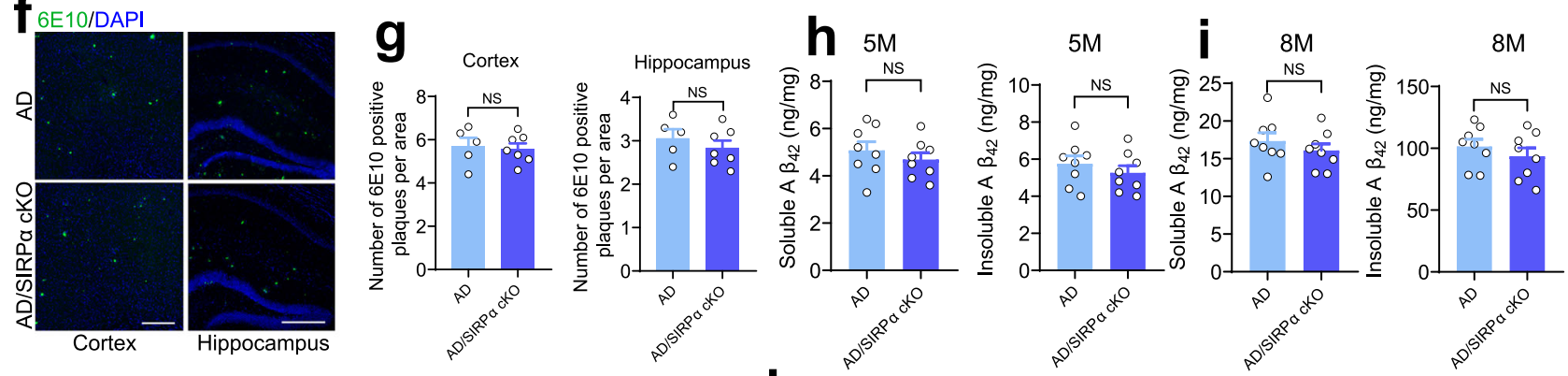

Iba-1/6E10
Diameter 20 50 $\mu \mathrm{m}$ plaque

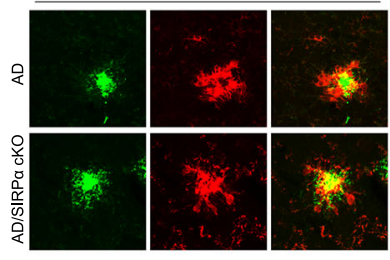

Diameter 50 80 $\mu \mathrm{m}$ plaque

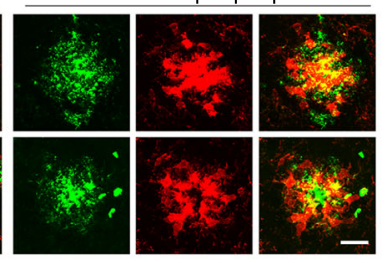

k
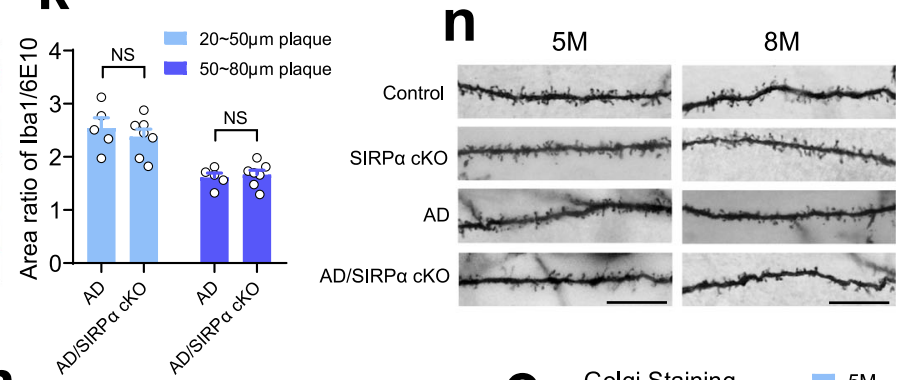

Cortex
PSD95/Synapsin
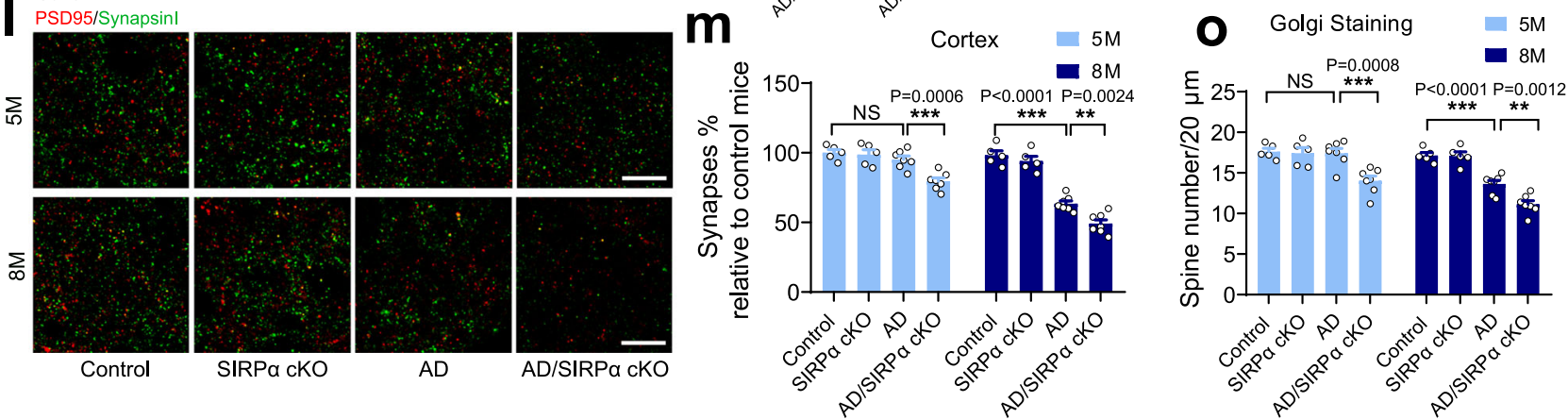

indicate that dysregulation of SIRPa-CD47 signal axis is involved in the inappropriate synaptic elimination in $\mathrm{AD}$ pathology.

\section{Discussion}

Microglia are of great significance in synaptic remodeling in central nervous system while the molecular mechanism under which is still under investigation ${ }^{23,24}$. Several studies have demonstrated that $\mathrm{iC} 3 \mathrm{~b} / \mathrm{C} 3 \mathrm{~b}-\mathrm{CR} 3$ signal positively regulates the removal of synapse during neurodevelopment ${ }^{3,25,26}$. Besides, there is evidence that neuronal CD47 can protect synapse from inappropriate elimination by microglia in visual system during early development-the first investigation of negative regulator in synaptic pruning in CNS, in which they considered SIRP $\alpha-C D 47$ signal axis plays an important role ${ }^{9}$. However, the exact effects of microglial SIRPa on synaptic remodeling in CNS remain obscure. Here we have demonstrated that microglial SIRPa-cKO mice have reduced synaptic number not only in dLGN but also in visual cortex as well as in hippocampus. Similar results were achieved in CD47-KO mice, indicating SIRPa-CD47 signal may act generally in negative regulation of synaptic remodeling in brain. We have provided evidence that microglia engulfed more 
Fig. 7 SIRP $\alpha$ deficiency enhanced cognitive impairment and synaptic loss without modifying A $\beta$ plaque depositions. a Schematics of the experimental

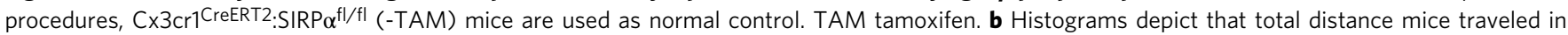
open filed experiment has no significant difference. $5 \mathrm{M}: n=13,14,15,15$ mice/group; $8 \mathrm{M}: n=10,12,10,12$ mice/group, one-way ANOVA, by Dunnett's multiple comparisons test. c Histograms depict escape latency of four different groups of mice in visible platform test (time to find the visible platform) before plaque deposition ( 5 months old) and after plaque deposition ( 8 months old). 5M: $n=13,14,15,15$ mice/group; 8 M: $n=10$, 12, 10,12 mice/group, one-way ANOVA, by Dunnett's multiple comparisons test. $\mathbf{d}$ Line charts show Morris Water Maze escape latency (time to find the hidden platform) in five consecutive days of four different genotypic mice at the age of 5 months old and 8 months old respectively. $5 \mathrm{M}: n=13,14,15,15$ mice/group; $8 \mathrm{M}: n=10$, 12, 10, 12 mice/group, two-way RM ANOVA, via Tukey's multiple comparisons test. e Histograms depict the time spent in target quadrant in Morris Water Maze probe trial of four different genotypic mice at the age of 5 months old or 8 months old. $5 \mathrm{M}: n=13,14,15,15$ mice/group, Kruskal-Wallis test via Dunn's multiple comparisons test; $8 \mathrm{M}: n=10,12,10,12$ mice/group, one-way ANOVA via Dunnett's multiple comparisons test. $\mathbf{f}$, $\mathbf{g}$ Representative immunostaining images exhibit $6 \mathrm{E} 10$ positive plaques in cortex and hippocampus of 8-months-old AD and AD/SIRP $\alpha$-cKO mice. Scale bar, $200 \mu \mathrm{m}$. Quantification analyses show that 6E10 positive plaques in AD and AD/SIRP $\alpha$-CKO mice were equivalent. $n=5,7$ mice/group; average of 5-6 fields from each mouse, two-tailed unpaired $t$-test. $\mathbf{h}, \mathbf{i}$ ELISA analyses of both soluble and insoluble $A \beta_{42}$ in 5-months-old and 8-months-old AD and AD/SIRP $\alpha$-cKO mice brain. $n=8$ mice/group, two-tailed unpaired $t$-test. j, $\mathbf{k}$ Representative images of 6 E10 immunoreactive plaques (diameter 20-50 $\mu \mathrm{m}$ as small groups; $50-80 \mu \mathrm{m}$ as big group) surrounded by microglia in 8-months-old AD and AD/SIRP $\alpha$ KO mice. Scale bar, $25 \mu \mathrm{m}$. Histograms show the area ratio of Iba- $1 /$ $6 \mathrm{E} 10$ are comparable in $\mathrm{AD}$ and $\mathrm{AD} / \mathrm{SIRP} \alpha-\mathrm{cKO}$ mice. $n=5,7$ mice/group. Average of 3-4 plaques from each mouse, two-tailed unpaired $t$-test. I, $\mathbf{m}$ Representative confocal images depict synaptic staining for presynaptic marker Synapsin I (green) and postsynaptic marker PSD95 (red) in cortex of 5months-old and 8-months-old mice. Synaptic number was determined as colocalized pre- and postsynaptic puncta. Scale bars, $10 \mu \mathrm{m} . n=5,5,7,7$ mice/ group, average of 10-12 fields from each mouse, one-way ANOVA via Dunnett's multiple comparisons test. $\mathbf{n}$, o Golgi staining images of apical dendritic spine in cortex of 5-months-old and 8-months-old mice. Scale bars, $20 \mu \mathrm{m} . n=5,5,7,7$ mice/group, average of 10 dendrites from each mouse; one-way ANOVA via Dunnett's multiple comparisons test. Data are mean \pm s.e.m. ${ }^{\star} P<0.05,{ }^{\star \star} P<0.01,{ }^{\star \star \star} P<0.001$, NS not significant. Detailed statistical information was listed in Supplementary Statistical Data. Source data are provided as a Source Data file.

synaptic structure in SIRPa cKO and CD47-KO mice during development. Besides, synaptic number was not altered in these mice at early stage of development (P5), indicating that microglia-mediated over-pruning may be involved in the synaptic reduction we observed. However, based on the results that number of synapses increases from P15 to P30, we cannot rule out the possibility that deficiency of SIRPa-CD47 signal affects synaptic formation, which may also contribute to the synaptic reduction at this duration.

It is reported that the $\mathrm{Cx} 3 \mathrm{cr} 1^{\mathrm{CreERT} 2}$ strain exhibits considerable leakiness. Van Hove et al. $^{27}$ analyzed tamoxifenindependent CreERT2 recombination, demonstrating $<3 \%$ leaky expression when the length of the floxed sequence is $>2.5 \mathrm{~kb}$. In our SIRPa-cKO model, the genetic distance between 2 loxP sites is $14.4 \mathrm{~kb}$, which shows little leakage effect without TAM. Deletion of SIRPa in microglia impacts its phagocytic ability, which leads to the excessive elimination of synaptic structures in SIRPa-cKO mice brain during early development. The phagocytic ability of microglia is not only the capacity to remove the functional-less waste but also the ability to recognize them. Maintaining brain homeostasis during development depends on the coordination of these two aspects ${ }^{28,29}$. Our data showed that SIRPa deficiency increased microglial phagocytosis towards synaptic structures. When co-incubated with mixed synaptosomes with/without CD47 expression, wild-type microglia preferred to engulf CD47 negative synaptosomes. In the meantime, SIRPa-deficient microglia exhibited impaired ability in discriminating CD47 signal by selecting both types of synaptosomes with no differences. In SIRPa-cKO mice, microglia engulfed more synaptic structures during early neurodevelopment. There was higher ratio of $\mathrm{CD} 47^{+} / \mathrm{PSD} 95^{+}$synaptic elements in SIRPacKO microglia compared to control, indicating these microglia failed to recognize CD47 signal (do not eat me signal) after losing SIRPa. Although microglial engulfment towards synapses in anesthetic mice increased as well, the ratio of $\mathrm{CD} 47^{+} / \mathrm{PSD} 5^{+}$ synaptic elements were decreased. This may be the result of decreased synaptic CD47 expression after neural activity inhibition, leaving more CD47 negative synaptic structures to be eliminated by microglia. These data indicate that the presence of SIRPa enables microglia to preserve synaptic structures by recognizing CD47 signal.
We have provided evidence that CD47 expresses differentially in synapses/synaptosomes. Inhibiting neural activity significantly decreases neuronal CD47 expression both in vitro and in vivo. Additionally, isoflurane treatment resulted in remarkable reduction of CD47 level in synaptosomes isolated from newborn mice, which is consistent with the previous report that CD47 is regulated by neural activity. Those isolated synaptosomes were easier to be engulfed by microglia, indicating that those less active synapses may be eliminated by microglia due to the loss of SIRPa-CD47 signal ${ }^{9}$. Interestingly, Toth et al. ${ }^{30}$ have observed that synaptic density is decreased in hippocampus of SIRPa KO mice (generated by crossing SIRPa floxP mice with Actin-Cre line), in which they considered it as a result of inhibited synapse maturation due to the loss of neuronal SIRPa. Here, we provide evidence that loss of microglial SIRPa may also contribute to the reduced synaptic density during early neurodevelopment. We have demonstrated that microglial SIRPa deletion in newborn mice increased synaptic elimination during pruning period, which showed persistent effects even in P30 mice. In our AD study, we induced microglial SIRPa ablation in two-months old mice (after the peak of pruning period) to exclude the potential impact of early SIRPa deficiency. Adult microglial SIRPa deficiency can no longer affect early synaptic pruning and shows little impact on synaptic density at later stages in normal condition. Consistently, those SIRPa-cKO mice did not show any behavioral changes as well. It seems that SIRPa-CD47 axis, as the negative regulatory signal, modulates the synaptic remodeling in a passive manner during neurodevelopment. SIRPa deficiency just releases the brake of microglia, it takes effect only when the synaptic elimination process is initiated.

In addition to the physiological conditions, positive signals that modulate microglia-mediated synaptic removal also play critical roles in some disease-related neuropsychiatric symptoms. For instance, $\mathrm{C} 1 \mathrm{q}$ level is aberrantly upregulated in neuronal synapses of AD model, which resulted in microglia-mediated early synaptic loss ${ }^{18}$. Blocking the C1q-dependent iC3b/C3b-CR3 signaling pathway can significantly alleviate memory loss in $\mathrm{AD}$ mice ${ }^{15}$. Similar complementary pathways are also activated in West Nile Virus infection, lupus or schizophrenia, which may further lead to dysregulated synaptic removal and significant neurological symptoms ${ }^{16,20,31}$. Besides, abnormal microglia derived from 


\section{a}

b

Control: $\mathrm{Cx} 3 \mathrm{Cr} 1^{\text {CreeRT2:SIRPa }}{ }^{\text {fllit }}(-\mathrm{TAM})$ SIRPa CKO: CX3cr1 ${ }^{\text {CreERT2:SIRPa }}{ }^{\text {R/I }}(+$ TAM 2M)
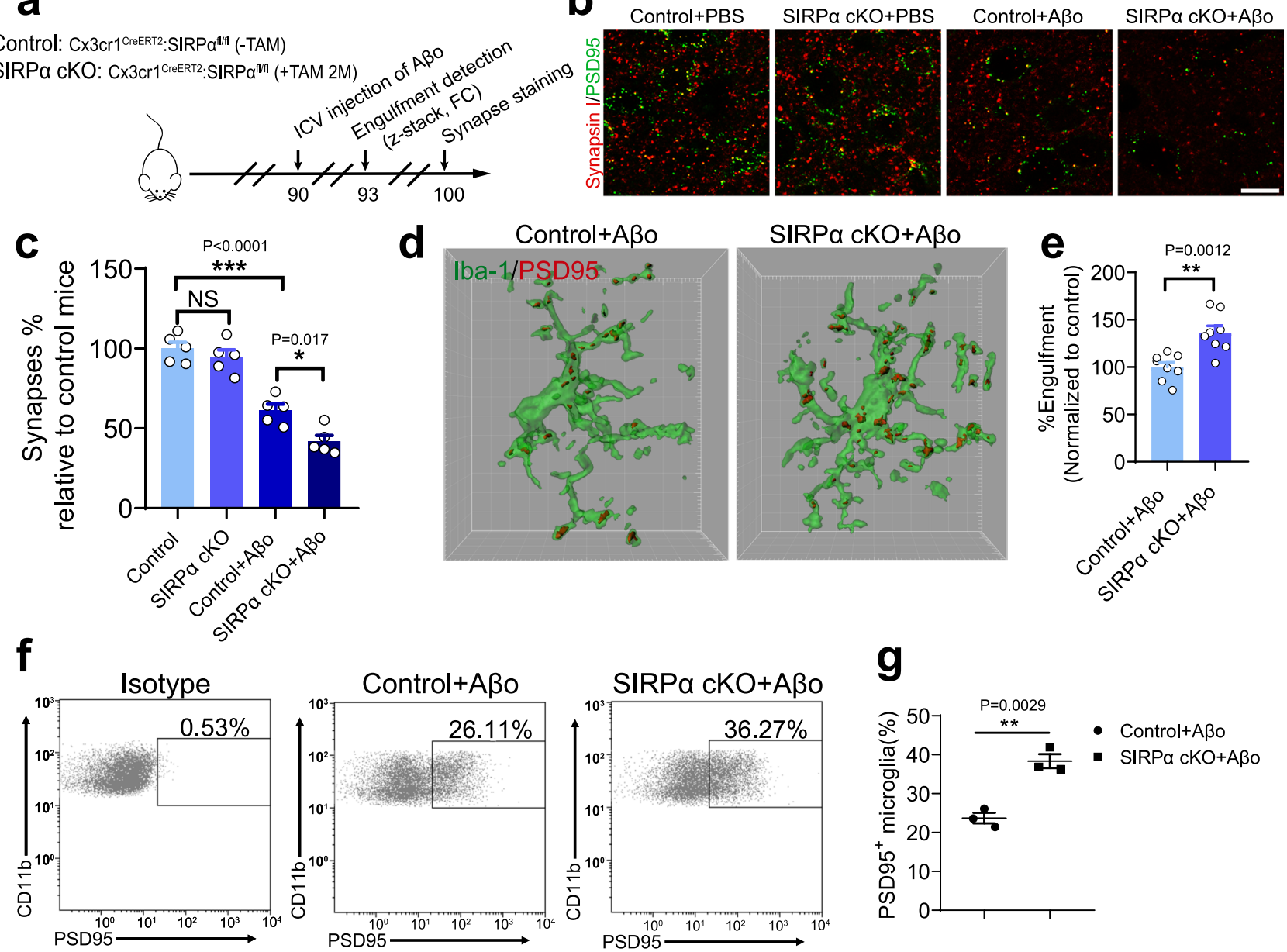

Fig. 8 Inhibition of microglial SIRPo increased A $\beta$ o-induced synapse loss by promoting microglia-mediated synaptic elimination. a Schematics of the

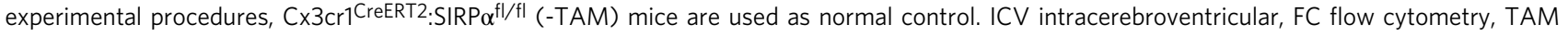
tamoxifen. b, c Representative confocal images (b) depict synaptic staining for presynaptic marker Synapsin I (red) and postsynaptic marker PSD95 (green) in cortex, Scale bars, $10 \mu \mathrm{m}$. $A \beta \mathrm{m}, A \beta_{42}$ monomer; $A \beta \mathrm{O}, A \beta_{42}$ oligomer. The histogram $\mathbf{c}$ displays the quantification of synaptic density in these mice. $n=5$ mice/group (male, 3 months age), average of 6-8 fields from each mouse. One-way ANOVA, by Tukey's multiple comparison test. d, e 3D reconstruction and surface rendering demonstrate larger volumes of PSD95 $5^{+}$puncta inside lba- $1^{+}$microglia in cortex from SIRP $\alpha$-cKO mice versus control mice after $A \beta 0$ stimulation ( $2 \mu \mathrm{g} /$ mouse), Grid line increments $=5 \mu \mathrm{m} . n=8$ mice/group, average of $8-9$ microglia from each mouse, two-tailed unpaired $t$ test. $\mathbf{f}, \mathbf{g}$ Flow cytometry analysis of synaptic material (PSD95 ${ }^{+}$) engulfed by microglia in SIRP $\alpha$-cKO or control mice after A $\beta 0$ injection. $n=3$ mice/ group, average of three tests from each mouse, two-tailed unpaired $t$ test. Data are mean \pm s.e.m. ${ }^{\star} P<0.05,{ }^{\star \star} P<0.01,{ }^{\star \star \star} P<0.001, N S$ not significant. Detailed statistical information was listed in Supplementary Statistical Data. Source data are provided as a Source Data file.

schizophrenia patient showed increased phagocytosis of synaptic structure, indicating microglial dysfunction contributes to the aberrant synaptic elimination during these disease progression ${ }^{6}$. Nevertheless, the role of negative regulatory signals of synaptic elimination in neurological disorder is largely unknown. In the present study, we have demonstrated that microglial SIRPa expression is downregulated during $\mathrm{AD}$ progression while SIRP $\beta 1$, which shares similar extramembrane domain with SIRP $\alpha$ but has a very different intracellular domain, is elevated both in human $\mathrm{AD}$ patients and $\mathrm{AD}$ mice brain ${ }^{32,33}$. All these changes indicate the increased phagocytic activity of microglia in $\mathrm{AD}^{32}$. While SIRP $\beta 1$ induces phagocytosis towards cell debris and $A \beta$ that is beneficial to the disease progression, our results show that downregulation of SIRPa enhances phagocytic activity towards synaptic elements that has detrimental effects. Whether microglial phagocytosis has double-edged effects or they are separately under control by different signal pathway needs further investigation. Downregulation of microglial SIRPa may result from the inhibition by $A \beta$ o generated in the early stage of the disease. $A \beta o$ can induce pro-inflammatory phenotype of microglia and there is evidence that microglial SIRPa is reduced during aging or inflammatory stress, indicating that chronic inflammation may contribute to $\mathrm{A} \beta \mathrm{o}$-induced SIRPa reduction in microglia ${ }^{22}$. These data together suggest the involvement of microglial SIRPa signal in $\mathrm{AD}$ as well as other aging related neurodegenerative diseases.

Using AD/SIRPa-cKO mice in which microglial SIRPa was specifically ablated at 2 months age, we can exclude the potential impact of neuronal SIRPa deficiency as well as the developmental change caused by early microglial SIRPa deletion. We found microglial SIRPa deficiency had little effect on the amyloid deposition, while it increased synapses loss and enhanced memory impairment in $\mathrm{AD}$ mice. Although phagocytic capability of SIRPa deficient microglia is enhanced, the ability of target recognition is compromised due to the blockage of SIRPa-CD47 signal. Moreover, the recruitment of microglia around the plaque was comparable between $\mathrm{AD} / \mathrm{SIRPa}-\mathrm{cKO}$ mice and $\mathrm{AD}$ control. Therefore, the overall effects of SIRPa deficiency on amyloid deposition are insignificant. We observed reduced synaptic 
density in SIRPa-cKO mice followed with A $\beta$ o administration. 3D imaging revealed that SIRP $\alpha$ deficient microglia engulfed more synaptic structures in $A \beta$ mice brain. In these models, $A \beta$ acted as an initial insult that triggers neurotoxic cascade. In addition, it also induces microglia-mediated synaptic loss by activating C1q-dependent iC3b/C3b-CR3 signaling pathway ${ }^{18}$. Our results suggest that lack of microglial SIRPa signal may further exacerbate the situation. Notably, ablation of microglial SIRP $\alpha$ at 2 months old shows little impact on synaptic density in non-AD mice while it induces excessive synaptic elimination in $\mathrm{AD}$ mice at later stages. These data suggest SIRPa signal modulates the synaptic removal in a passive manner when synaptic elimination process is initiated by $\mathrm{A} \beta$ during $\mathrm{AD}$ pathology. Overexpression of SIRP $\alpha$ in $A \beta$ stimulated microglia can prevent its excessive phagocytosis towards synaptosomes. Indeed, these results would indicate that SIRP $\alpha-C D 47$ signal axis may be a potential target in synaptopathology of AD.

$\mathrm{CD} 47$ expression is also downregulated during AD progression. In $\mathrm{AD}$ mice, synaptosomal $\mathrm{CD} 47$ level decreases at 8 months age. The declination of $\mathrm{CD} 47$ expression at this stage may be the results of long-term decreased neural activity ${ }^{34}$, as in anesthetized mice, we also observed CD47 downregulation in synaptic structure. Notably, there was evidence that microglial surveillance is significantly increased in anesthetized mice model, indicating the potential increase of microglia-mediated synaptic clearance ${ }^{35,36}$. Furthermore, synaptosomes isolated from late stage of $\mathrm{AD}$ mice brain $(8 \mathrm{M})$ with lower $\mathrm{CD} 47$ expression are easier to be engulfed by microglia. CD47-KO mice phenocopy the enhanced loss of synapses and microglial engulfment in SIRPacKO mice followed with ICV injection of $A \beta \mathrm{o}$, suggesting that SIRP $\alpha$ and CD47 may work together in the synaptic pathology of $\mathrm{AD}$. The dysregulated synaptic elimination is evident in $\mathrm{AD}$ pathology, with the previous reports revealing neuronal abnormality, in which there was enhanced $\mathrm{Clq}$ expression in synapses ${ }^{18}$. Together, our findings provide evidence that microglial SIRPa signal is inhibited in AD, which significantly accelerates synapses loss by promoting inappropriate synaptic elimination during $\mathrm{AD}$ progression. We have demonstrated that such negative regulating signal of synaptic pruning/elimination is dysregulated in neurological diseases, which further contributes to the synaptopathology and neurological symptoms. Since SIRPa-CD47 signal has been widely studied as immune checkpoint pathway, it may also be a potential therapeutic target in synaptopathology related neurological disorders ${ }^{37,38}$.

In summary, our findings provide evidence that microglial SIRPa signal negatively regulate synaptic pruning during early neurodevelopment, which is important for maintaining homeostasis in brain. During AD pathology, microglial SIRPa signal is disrupted, which subsequently induces excessive elimination of synapses and enhances cognitive impairment.

\section{Methods}

Mice and treatment. All experimental procedures were approved by Model Animal Research Center of Nanjing University and according with Laboratory Animal Care Guidelines. C57BL/6 mice, Cx3cr1 CreERT2 mice, CD47-KO mice, and $\mathrm{AD}$ (APPswe and PSEN1dE9 in a single locus) mice were purchased from the Model Animal Research Center of Nanjing University.

$\mathrm{SIRP} \alpha \mathrm{fl} / \mathrm{fl}$ mice were generated by flanking sirpa exon 2-6 with loxP sites. The base pair length between the 2 lox P sites was $14446 \mathrm{bp}$. Microglial SIRPa-cKO mice were generated after we treated $\mathrm{Cx} 3 \mathrm{cr} 1^{\mathrm{CreERT}}$ : $\mathrm{SIRPa}{ }^{\mathrm{fl} / \mathrm{fl}}$ mice with tamoxifen (TAM) at different stages indicated. After TAM induction, PCR genotyping generated a 343-bp fragment suggesting SIRPa gene (exon 2-6) ablation. While before exon 2-6 deletion, the genetic distance between these two primers is $\sim 14 \mathrm{~kb}$, resulting in no bands under normal PCR condition (elongation time is $45 \mathrm{~s}$ ). All primers used for mice genotyping were listed in Supplementary Table 1. All mice were bred under standard conditions of constant temperature $\left(22 \pm 1^{\circ} \mathrm{C}\right)$, humidity (relative, $30 \%$ ), in a pathogen-free facility and exposed to a 12 -h light/dark cycle.
}

Tamoxifen injection. In brief, tamoxifen was dissolved in ethanol then diluted by corn oil. Neonatal mice were injected with $50 \mu \mathrm{g}$ tamoxifen solution $(1 \mu \mathrm{g} / \mu \mathrm{l})$ or vehicle into stomach at $\mathrm{P} 1, \mathrm{P} 2$, and $\mathrm{P} 3^{39}$. Adult mice were injected with tamoxifen intraperitoneally once every $24 \mathrm{~h}$ for a total of 5 consecutive days, with the dose of $75 \mathrm{mg}$ tamoxifen/kg body weight $(20 \mu \mathrm{g} / \mu \mathrm{l})$. Mice were killed for further investigation at several time points as indicated.

Isoflurane treatment. P7/P28 mice were exposed to $100 \%$ oxygen carrying $1.5 \%$ isoflurane for 4 hours. Oxygen was delivered at $5 \mathrm{~L} / \mathrm{min}$ flow rate controlled by a calibrated flowmeter. In control group, mice were exposed to room air for $4 \mathrm{~h}$. All mice were returned to their original cages together upon regaining righting reflex. One day after isoflurane treatment, mice were euthanatized and brains were taken out for further analysis.

Stereotaxic injections of $A \beta$. For ICV injection, 3 months aged mice (male) were anesthetized and $2 \mu \mathrm{l} A \beta$ oligomers $(1 \mu \mathrm{g} / \mu \mathrm{l})$ were injected into the left lateral ventricles. The stereotaxic coordinates were $0.4 \mathrm{~mm}$ posterior to the Bregma, $1 \mathrm{~mm}$ lateral to the midline, and $2.5 \mathrm{~mm}$ ventral to the surface of the dura mater. PBS containing 4\% DMSO was injected as control. Mice were killed for further analysis at different time points indicated.

\section{Cell cultures and treatment}

Primary neuron culture. Briefly, cerebral cortices were harvested from E14-E16 embryos. The meninges were removed and the cerebral cortices were dissected into small pieces and then digested by $0.025 \%$ trypsin for $30 \mathrm{~min}$ at $37^{\circ} \mathrm{C}$. Cell suspension were filtered through cell strainer to remove chunks. After centrifugation, cells were plated onto PDL-coated plastic coverslips in 24-well plates for neuronmicroglia co-culture, or cells were plated onto PDL-coated plastic coverslips in 6 well plates for protein extraction. The cells were maintained in Neurobasal medium (Invitrogen, Camarillo, CA, USA) with B27 supplement (Invitrogen, Camarillo, CA, USA) and GlutaMax Supplement (Invitrogen, Camarillo, CA, USA) for 14 days before neuron-microglia co-culture. Neurons were cultured for 10-12 days and incubated with TTX $(1 \mu \mathrm{M})$ for $48 \mathrm{~h}$ before immunofluorescent staining or protein extraction. For CD47 expression assay, neurons were stimulated with $0.2 \mu \mathrm{M} A \beta$ monomer or $\mathrm{A} \beta$ oligomer for $24 \mathrm{~h}$ before protein analysis.

Primary microglial culture. Briefly, cerebral cortices were harvested from neonatal mice before digestion and centrifugation to obtain a pellet for cell seeding. The microglia were harvested from the astrocyte layer 6-10 days later by shaking the flasks at $200 \mathrm{rpm}$ for $1-2 \mathrm{~h}$ at $37^{\circ} \mathrm{C}$. The media was centrifuged and the cells were pelleted. Cells were seeded onto poly-D-lysine (PDL)-coated plastic coverslips in 24 -well plates $\left(1 \times 10^{5}\right.$ cells/per well) with DMEM/F12 complete medium plus $10 \%$ heat-inactivated fetal bovine serum, or the cell suspension were added into neurons directly for subsequent neuron-microglia co-culture.

For lentiviral transduction, EFla-SIRPa lentivirus expressing system (LV6, $1 \times 10^{9} \mathrm{TU} / \mathrm{ml}$, Genepharma, Shanghai, China) was used to overexpress SIRPa in isolated microglia. Cells were exposed to lentivirus (at a multiplicity of infection of five) 3 days after seeding, and incubated for $6 \mathrm{~h}$ before the medium was refreshed. Two days later cells were subjected to $A \beta$ stimulation.

Microglia were stimulated with $0.2 \mu \mathrm{M}$ A $\beta$ monomer or $\mathrm{A} \beta$ oligomer for $24 \mathrm{~h}$ before protein analysis. For phagocytic assay, cells were subjected to $0.2 \mu \mathrm{M} A \beta 42$ treatment for $3 \mathrm{~h}$ before incubating with synaptosomes.

Neuron-microglia co-cultures. Briefly, primary cortical microglia were harvested from the astrocyte layer by shaking the flasks at $200 \mathrm{rpm}$ for $1-2 \mathrm{~h}$ at $37^{\circ} \mathrm{C}$, isolated microglia were added into DIV 14 neurons at a $1: 3$ microglia $\left(6.6 \times 10^{3}\right.$ cells/per well) to neuron $\left(2 \times 10^{4}\right.$ cells/per well $)$ ratio for 3 days co-culture. Co-cultures were terminated by fixing cells with $4 \%$ PFA for further analysis.

Immunoblotting. To obtain total proteins, brain tissues or cultured cells were lysed by RIPA lysis buffer (Thermo Scientific, Rockford, IL, USA) supplemented with protease inhibitor, and protein concentration was quantified by BCA protein assay kit (Thermo Scientific, Rockford, IL, USA). The protein samples were loaded onto $10 \%$ SDS/PAGE gel before transferred onto poly vinylidene difluoride (PVDF) membranes (Roche Diagnostics, Indianapolis, IN, USA). Then 5\% nonfat milk was used to block the membranes before incubation with different primary antibodies overnight at $4{ }^{\circ} \mathrm{C}$ Primary antibodies were used as follows: goat anti-CD47 (1:1000, R\&D Systems, AF1866), rabbit anti-SIRPa (1:5000, Abcam, ab8120), rabbit anti SIRPB1 (1:2000, LifeSpan Biosciences, LS-C679465), and mouse anti-GAPDH (1:2000, Santa Cruz Biotechnology, sc-59541). After washing, the membranes were incubated with horseradish peroxidase (HRP)-conjugated secondary antibodies for $1 \mathrm{~h}$. Secondary antibodies were used as follows: donkey anti-rabbit IgG-HRP (1:1000-1:5000, Santa Cruz Biotechnology, sc-2077); donkey anti-mouse IgG-HRP (1:2000-1:5000, Santa Cruz Biotechnology, sc-2314); donkey anti-goat IgG-HRP (1:1000-1:2000, Santa Cruz Biotechnology, sc-2020). Then ECL Western Blotting Detection Kit (Thermo Scientific) was used for color detection. The quantification of protein level was analyzed by ImageJ analysis software. Uncropped gel scans are provided in a Source Data file. 
Immunofluorescence staining. Mouse brains were collected after transcardial perfusion with phosphate-buffered saline (PBS) and 4\% paraformaldehyde (PFA) sequentially. Brains were fixed in $4 \%$ PFA for $24 \mathrm{~h}$, then soaked in $15 \%$ and $30 \%$ sucrose solution for dehydration. Coronal sections of $20 \mu \mathrm{m}$ or $30 \mu \mathrm{m}$ thickness were cut in a Leica cryostat (Leica CM1950) for immunofluorescent staining. Afterwards, tissue sections were incubated in 5\% bovine serum albumin (BSA) with $0.3 \%$ triton-X 100 in PBS for $1 \mathrm{~h}$. The brain sections were incubated with primary antibodies at $4{ }^{\circ} \mathrm{C}$ overnight, and then washed three times with PBS before incubated with the appropriate secondary fluorescent antibodies for $1 \mathrm{~h}$ at room temperature. Tissue was stained with DAPI and rinsed three times with PBS before image capture using Leica TCS SP8-MaiTai MP confocal microscope. For primary cultured cell immunostaining, cells were fixed with $4 \%$ PFA for $10 \mathrm{~min}$, and the following procedure were the same as brain tissues.

Information of antibodies used were as follows: primary antibodies: rabbit anti Iba-1 (1:500, wako, 019-19741); chicken anti-MAP2 (1:1000, Abcam, ab5392); chicken anti-GFAP (1:1000, Abcam, ab4674); mouse anti-SIRPa (1:500, Millipore, MAB1407P); rabbit anti-NeuN (1:1000, Abcam, ab177487); rabbit anti-Homer1 (1:200, Synaptic Systems, 160-003); guinea pig anti-Vglut1 (1:1000, Millipore, AB5905); guinea pig anti-Vglut2 (1:1000, Millipore, AB2251); mouse anti-PSD95 (1:100-1:500, Abcam, ab2723); rabbit anti-Synapsin 1 (1:1000, Millipore, AB1543); mouse anti-Synaptophysin (1:2000, Sigma, S5768); rat anti-CD47(1:200, BD Pharmingen, 555297); and 6E10 (1:500, BioLegend, 803001). Secondary fluorescent antibodies (Thermo Fisher Scientific, 1:1000) were as follows: Goat anti-Rabbit IgG $(\mathrm{H}+\mathrm{L})$ Secondary Antibody, Alexa Fluor 488 conjugate (Thermo Fisher Scientific, A-11034); Goat Anti-Rabbit IgG (H + L) Antibody, Alexa Fluor 594 Conjugated (Thermo Fisher Scientific, A-11012); Goat anti-Mouse IgG (H + L) Cross-Adsorbed Secondary Antibody, Alexa Fluor 488 (Thermo Fisher Scientific, A-11001); Goat anti-Chicken IgY $(\mathrm{H}+\mathrm{L})$ Secondary Antibody, Alexa Fluor 488 (Thermo Fisher Scientific, A-11039); Goat anti-Rabbit IgG (H + L) Secondary Antibody, Alexa Fluor 633 (Thermo Fisher Scientific, A-21071); Donkey Anti-Rat IgG (H + L) Antibody, Alexa Fluor 594 Conjugated (Thermo Fisher Scientific, A-21209); Goat Anti-Guinea Pig IgG $(\mathrm{H}+\mathrm{L})$ Antibody, Alexa Fluor 488 Conjugated (Thermo Fisher Scientific, A-11073); Goat anti-Mouse IgG (H + L) Cross-Adsorbed Secondary Antibody, Alexa Fluor 594 (Thermo Fisher Scientific, A-11005); Goat anti-Chicken IgY $(\mathrm{H}+\mathrm{L})$ Secondary Antibody, Alexa Fluor 594 (Thermo Fisher Scientific, A-11042); Donkey anti-Rat IgG (H + L) Cross-Adsorbed Secondary Antibody, Alexa Fluor 488 (Thermo Fisher Scientific, A-21208).

TUNEL staining was performed using TUNEL BrightGreen Apoptosis Detection Kit (A112, Vazyme) according to protocols.

Eye-specific segregation analysis. Mice were anesthetized with isoflurane, for injection of neonatal mice, a pair of small scissors was used to open the eyelid to expose the sclera. Afterwards, a sterile $30.5 \mathrm{G}$ needle was used to puncture a small hole in the eye where the sclera begins. Once the vitreous flowed out of the hole, a blunt ended needle attached to a Hamilton syringe was inserted into the hole to inject dye into the eye slowly. Cholera toxin subunit B (CTB) conjugated to Alexa 594 (ThermoFisher Scientific, C-22842) and Alexa 647 (ThermoFisher Scientific, C-34778) were intraocularly injected in the right and left eye (1-2 $\mu \mathrm{l} ; 0.5 \%$ in sterile saline) respectively ${ }^{40}$. Mice were killed $24 \mathrm{~h}$ later and brain tissues were fixed in $4 \%$ PFA overnight, cryoprotected in $15 \%$ and $30 \%$ sucrose sequentially, and then sectioned coronally at $40 \mu \mathrm{m}$ using Leica cryostat (Leica CM1950).

Images were acquired by Leica TCS-SP8 Laser Scanning microscope with $\times 10$ objective, and images were analyzed by ImageJ software using the multi-threshold quantitative method ${ }^{40}$. In each genotype 7 mice were examined and at least four images were analyzed per mouse. The degree of overlap was analyzed by unpaired $t$ test at every threshold level.

Whole-cell patch clamp recordings on brain slices. Mice were decapitated after sodium pentobarbital anesthesia, the coronal slices $(300 \mu \mathrm{m}$ thickness) containing hippocampus were obtained by a vibroslicer (VT 1200S, Leica, Wetzlar, Germany) and incubated in artificial cerebrospinal fluid (ACSF, NaCl $125 \mathrm{mM}, \mathrm{KCl} 3.25 \mathrm{mM}$, $\mathrm{NaH}_{2} \mathrm{PO}_{4} 1.25 \mathrm{mM}, \mathrm{NaHCO}_{3} 25 \mathrm{mM}, \mathrm{MgCl}_{2} \cdot 6 \mathrm{H}_{2} \mathrm{O} 1 \mathrm{mM}$, D-glucose $11 \mathrm{mM}$, $\mathrm{CaCl}_{2} 2 \mathrm{mM}$ ) with $95 \% \mathrm{O}_{2}$ and $5 \% \mathrm{CO}_{2}$, at $32 \pm 0.5{ }^{\circ} \mathrm{C}$ for at least $1 \mathrm{~h}$. Then the slices were maintained at room temperature. For whole-cell patch clamp recordings, the slices were transferred to a submerged chamber and continuously perfused with $95 \% \mathrm{O}_{2}$ and $5 \% \mathrm{CO}_{2}$ oxygenated ACSF at a rate of $2 \mathrm{~mL} / \mathrm{min}$ maintained at $32 \pm 0.5^{\circ} \mathrm{C}$.

Whole-cell patch clamp recordings were performed with borosilicate glass pipettes (resistance of 4-6 $\mathrm{M} \Omega$ ) filled with internal solution (140 $\mathrm{mM} \mathrm{K}$ methylsulfate, $7 \mathrm{mM} \mathrm{KCl}, 2 \mathrm{mM} \mathrm{MgCl} 2,10 \mathrm{mM}$ HEPES, $0.1 \mathrm{mM}$ EGTA, $4 \mathrm{mM}$ $\mathrm{Na}_{2}$-ATP, $0.4 \mathrm{mM}$ GTP-Tris, adjusted to $\mathrm{pH} 7.25$ by $\mathrm{KOH}$ ). The hippocampal CA1 neurons were visualized through Olympus BX51WI microscope (Olympus, Tokyo, Japan) during recording. mEPSCs were recorded at a holding potential of $-70 \mathrm{mV}$ with the presence of $1 \mu \mathrm{M}$ tetrodotoxin (MedChen Express) and $50 \mathrm{mM}$ gabazine (GABAA receptor antagonist; Tocris, Bristol, UK) for at least $1 \mathrm{~h}$ through Axopatch-700B amplifier (Axon Instruments, Foster City, CA), and the signals were transferred into a computer through a Digidata-1440A (Axon Instruments, Foster City, CA) for data capture. Clampex 10.7 (Axon Instruments, Foster City, CA) was used for data collection and pClamp10.0 (Axon Instruments, Foster City, $\mathrm{CA})$ was used for data analysis.
Synaptosome isolation and pHrodo labeling. Briefly, mice were anesthetized and forebrains were quickly removed and homogenized in ice-cold gradient buffer (320.0 $\mathrm{mM}$ sucrose, $5.0 \mathrm{mM}$ HEPES, $0.1 \mathrm{mM}$ EDTA, $\mathrm{pH}$ 7.5). The homogenate was centrifuged at $1000 \times g$ for 20 min to collect supernatant, and supernatant was centrifuged at $1200 \times g$ for $10 \mathrm{~min}$ to discard cell nuclei and debris and collect supernatant. The supernatant was centrifuged at $10,000 \times g$ for $10 \mathrm{~min}$ to obtain pellet. The pellet was resuspended in gradient buffer and loaded onto a sucrose gradient $(0.8 \mathrm{M}: 1.2 \mathrm{M}=1$ : 1). The thin layer (the layer between $0.8 \mathrm{M}$ sucrose and $1.2 \mathrm{M}$ sucrose) was collected carefully after centrifugation at $100,000 \times g$ for $1 \mathrm{~h}$, and diluted with an equal volume of ultrapure water before centrifuged at $100,000 \times g$ again to acquire the purified synaptosome pellet. The synaptosome pellet was resuspended in ice-cold artificial cerebrospinal fluid (aCSF, $2 \mathrm{mM} \mathrm{CaCl}_{2}, 132 \mathrm{mM} \mathrm{NaCl}, 3 \mathrm{mM} \mathrm{KCl}, 2 \mathrm{mM} \mathrm{MgSO}_{4}$, $1.2 \mathrm{mM} \mathrm{NaH}_{2} \mathrm{PO}_{4}, 10 \mathrm{mM}$ HEPES, and $10 \mathrm{mM}$ glucose, $\mathrm{pH}$ 7.4).

For pHrodo labeling, synaptosomes were incubated with pHrodo Red succinimidyl (NHS) ester (Life Technologies, P36000) or pHrodo Green STP ester (Life Technologies, P36013) in sodium carbonate buffer $\mathrm{pH} 9.0$ for $2 \mathrm{~h}$ at $4{ }^{\circ} \mathrm{C}$ in the dark at the concentration of $1 \mu \mathrm{L}$ pHrodo/1 mg synaptosomes. After incubation unconjugated pHrodo was washed by DPBS.

In vitro microglial engulfment assay. Equal amount of WT or CD47-KO pHrodo-red conjugated synaptosomes were added to cultured WT or SIRPa KO microglia. After incubation period of $1 \mathrm{~h}$ with synaptosomes, cells were washed in warm $\left(37^{\circ} \mathrm{C}\right) \mathrm{PBS}$ and fixed in warm $4 \%$ PFA for $10 \mathrm{~min}$. After fixation, cells were washed in PBS again, then blocked and permeabilized in $5 \%$ bovine serum albumin (BSA) and $0.3 \%$ triton-X100 in PBS for $1 \mathrm{~h}$ at RT. Cells were incubated with primary antibody overnight at $4{ }^{\circ} \mathrm{C}$ and followed by secondary fluorescent antibody staining for $1 \mathrm{~h}$ at RT. Cells was staining with DAPI and rinsed three times with PBS before image capture using Leica TCS SP8-MaiTai MP confocal microscope. In all, 5-6 fields of view were collected randomly from each coverslip. When WT or CD47-KO synaptosomes were conjugated to pHrodo-red or pHrodo-green dyes respectively, images should be captured when cells were alive because fluorescence of pHrodo-green is undetectable after fixation. An equal amount of pHrodo-red and green labeled synaptosomes were mixed and added to primary cultured microglia by the amount of $0.017 \mathrm{mg}$ of total synaptosomes. Cells were washed in warm $\left(37^{\circ} \mathrm{C}\right) \mathrm{PBS}$ after $1 \mathrm{~h}$ of incubation with synaptosomes, then stained live for $15 \mathrm{~min}$ at $37^{\circ} \mathrm{C}$ with CX3CR1 antibody conjugated to Alexa Fluor 647 (1:100, BioLegend, 149004). Cells were imaged live using Leica TCS SP8-MaiTai MP confocal microscope using LAS X 3.3.0 software.

CD47 positive synaptic structures labeling in vivo. For Vglut1/CD47 or PSD95/ CD47 double labeling in brain section, $\mathrm{z}$-stack images (at $0.2 \mu \mathrm{m}$ intervals, 12 images) were imaged using a Zeiss LSM 880 microscope with a $\times 63$ objective and $2 \times$ electronic zoom.

For PSD95/CD47/Iba-1 triple labeling in microglia in vivo, z-stack images (at $0.3 \mu \mathrm{m}$ intervals) were imaged using a Zeiss LSM 880 microscope with a $\times 63$ objective and $2 \times$ electronic zoom. In all, 3D-structured illumination images were generated using ZEN 3.1 blue edition software (Zeiss).

NanoSight detection. Synaptosomes were incubated with anti-CD47-FITC antibody (1:100, BD Pharmingen, 555298) on ice for $30 \mathrm{~min}$ in the dark before detection. Then synaptosomes extracted from mice forebrain were analyzed using NanoSight NS300 (Malvern Instruments) to obtain particle diameter data, particle number and FITC fluorescent information through NanoSight NTA 3.2 software (Malvern Instruments).

Preparation of single-cell suspension and flow cytometry. Mice were anesthetic and executed to harvest cerebrums. Cerebrums were dissected into small pieces and digested in papain for $30 \mathrm{~min}$. After digestion, cell suspensions were filtered through $70 \mu \mathrm{m}$ cell strainer to remove clumps, and cell pellets were resuspended in $30 \%$ Percoll solution following centrifugation. The upper myelin layer was discarded, and cell pellets were resuspended in medium (HBSS containing 2\% FBS and $1 \mathrm{mM}$ EDTA) to obtain single-cell suspension. Isolated single cells were treated as follows: 15 min incubation with FC-receptor blocker CD16/CD32 antibody (Thermo Scientific, MA5-18012, 1:200) at $4{ }^{\circ} \mathrm{C}$, followed by $30 \mathrm{~min}$ incubation with anti-CD45-PE antibody (eBioscience, 12-0451-82, 1:200), anti-SIRPa-FITC antibody (Biolegend, 144006, 1:200), and anti-CD11b-APC antibody (eBioscience, 170112-82, 1:200) or rat IgG isotype. For intracellular staining, cells were fixed with $4 \%$ PFA for 10 min and permeabilized with PBS containing $0.1 \%$ Triton-X 100 . Cells were incubated with anti-PSD95 antibody (Cell Signaling Technology, 3450, 1:500) for $30 \mathrm{~min}$, then incubated with secondary fluorescent antibodies for $30 \mathrm{~min}$ FACS analysis was performed on Beckman Coulter Gallios Flow Cytometer with Kaluza 1.0 software (Beckman Coulter). The data were analyzed by Kaluza Analysis 1.5a Software (Beckman Coulter).

Adult microglia isolation. Mice were anesthetized and perfused intracardially with ice-cold Dulbecco's phosphate-buffered saline to harvest brains. After the subsequent tissue dissociation, debris removal and red blood cell removal procedures, Adult Brain Dissociation Kit (130-107-677, Miltenyi Biotec) was used to generate single-cell suspension. Microglia were further isolated from the single-cell 
suspension using MACS Separation Columns (MS) (130-042-201, Miltenyi Biotec) and magnetic CD11b Microbeads (130-093-634, Miltenyi Biotec).

Stereotaxic injections of AAV. Mice were anesthetized and $100 \mathrm{nl}$ AAV (OBIO technology, $1.25 \times 10^{13} \mathrm{GC} / \mathrm{mL}$, AAV-hSyn-hM4D(Gi)-mCherry-WPRE) were injected into the primary visual cortex. The stereotaxic coordinates were $3.5 \mathrm{~mm}$ posterior to the Bregma, $2.6 \mathrm{~mm}$ lateral to the midline, and $0.5 \mathrm{~mm}$ ventral to the surface of the dura mater. AAV-hSyn-mCherry-P2A-3xFLAG-WPRE was injected as control. Four weeks later, mice were injected Clozapine-N-oxide (CNO) (Sigma, C0832) intraperitoneally at the dose of $0.5 \mathrm{mg} / \mathrm{kg}$ twice a day for consecutive 3 days. Mice were killed for further analysis $4 \mathrm{~h}$ after the final injection of CNO. For immunofluorescence, brain tissue was fixed in $4 \%$ PFA overnight, cryoprotected in $15 \%$ and $30 \%$ sucrose before sectioning.

A $\beta$ preparation. Briefly, $A \beta 1-42$ peptides were dissolved in hexafluoroisopropanol (HFIP) and was dried in an airing cupboard subsequently. $A \beta$ monomers prepared by dissolving the $A \beta$ in dimethyl sulfoxide (DMSO) at the concentration of $2.2 \mathrm{mM}$. A $\beta$ oligomers were prepared by dissolving $\mathrm{A} \beta$ in DMSO and oligomerized by incubating at room temperature for $48 \mathrm{~h}$. A $\beta$ oligomers were diluted at the concentration of $100 \mathrm{mM}$ (stock solution). $\mathrm{A} \beta$ monomers and $\mathrm{A} \beta$ oligomers were stored at $-80^{\circ} \mathrm{C}^{41}$.

A $\beta 42$ ELISA. Soluble $A \beta 42$ was directly obtained in brain homogenates prepared with ice-cold lysis buffer. To detect insoluble cerebral $A \beta 42$ in brain, insoluble pellets were further extracted in $70 \%$ formic acid by sonication and spun at $13,000 \times g$ for 20 min. Samples were neutralized in $1 \mathrm{M}$ Tris buffer. The levels of $\mathrm{A} \beta 42$ were quantified using commercially available human ELISA kits (Wako-296-64401) according to manufacturer's guidelines. Data obtained from the cortical homogenates were expressed as nanograms of $\mathrm{A} \beta$ content per milligrams of total protein ( $\mathrm{ng} / \mathrm{mg}$ ).

Golgi staining. Golgi staining was performed using FD Rapid GolgiStain ${ }^{\mathrm{TM}}$ kit according to its manufacturer's protocol (FD NeuroTechnologies, PK401, Columbia, $\mathrm{MD}$ ). Briefly, brains were immersed in impregnation solution (a mixture of FD Solution $\mathrm{A}: \mathrm{B}=1: 1)$ for 2 weeks at room temperature in the dark. Then brains were immersed in FD Solution $\mathrm{C}$ and preserved in the dark at room temperature for $48-72 \mathrm{~h}$. The brain tissues were coronally sliced $(150 \mu \mathrm{m})$ using Leica CM1950 cryostat and mounted on $0.5 \%$ gelatin-coated slides. The sections were then stained with the staining solution according to manufacturer's protocol and coverslipped using Permount medium. Images of cortical pyramidal neurons were captured using Olympus BX51 with Cell sens dimension 1.12 software (Olympus) at random.

For pyramidal neuron spine density analysis, the number of spines was counted on the following segments: for apical dendrites, dendritic segments $(20 \mu \mathrm{m})$ located further than $100 \mu \mathrm{m}$ from cell soma of pyramidal neurons were quantified.

\section{Behavioral analysis}

Open field. Mice were placed in the center of an empty field $(40 \times 40 \times 24 \mathrm{~cm})$ and allowed to explore freely. Total distance the mice traveled within 5 min was recorded. The maze was cleaned with $70 \% 2$-propanol between the trials.

Morris water maze. The procedures consisted of 1 day of the visible platform test and 5 days of the hidden platform test, plus a spatial probe trial. Time taken by the mouse to find and climb onto the platform was recorded as latency. In the probe trial, the percentage of total time in target quadrant was measured. Tracking of animal movement was achieved with a DigBehv-MM tracker system (MobileDatum Co. Ltd, Shanghai, China).

\section{Fluorescent images quantification}

Cell number and morphology. Cell density was quantified by $\mathrm{Iba}^{+} / \mathrm{NeuN}^{+} / \mathrm{MAP} 2^{+}$ cell number in a given area. Cell volume was determined as $\mathrm{Ibal}^{+} / \mathrm{NeuN}^{+}$area per cell. Primary cell purity was determined by MAP2 ${ }^{+}$cell/DAPI or $\mathrm{Ibal}^{+}$cell/DAPI Microglial branches in vivo were analyzed as follows. Brain sections stained with
Iba-1 were imaged using Leica TCS SP8-MaiTai MP to obtain z-stack images (at $1 \mu \mathrm{m}$ intervals). Images were performed $\mathrm{z}$-stack projection and converted into 8-bit. The modified images were binarized and skeletonized before branch analysis using ImageJ-Fiji 2.0.0 software ${ }^{22}$.Circularity of microglia in vitro was obtained by the formula: Circularity $=4 \pi$ (area/perimeter $\left.{ }^{2}\right)$. A circularity value of 1.0 indicates a perfectly circular cell, and values near zero indicate elongated and ramified microglia. All images were processes using ImageJ.

Synaptic density in vivo. Briefly, $20 \mu \mathrm{m}$ sections stained with synaptic markers (Homer1/Vglut1, Homer1/Vglut2, Synapsin1/PSD95) were captured by Leica TCS SP8-MaiTai MP confocal microscope or Zeiss LSM 880 microscope through x63 objective lens. Captured images were used to quantify the number of colocalized preand postsynaptic puncta by ImageJ software, and single-channel images were used to quantify single synaptic marker density by ImageJ. Synaptic density was determined as puncta number/given area. Researchers were blinded to genotype during imaging.

Synaptic density in vitro. Neuron-microglia co-cultures were quantified by immunofluorescent staining using antibodies for MAP2 (1:1000, Abcam, ab5392) synaptophysin (1:2000, Sigma, S5768), PSD95 (1:250-1:500, Abcam, ab2723), and Iba-1 (1:500, Wako, 019-19741) respectively. Microglia cells were centered for image capture by Leica TCS-SP8 Laser Scanning microscope with $\times 40$ objective. To quantify the density of synaptophysin or PSD95 puncta, concentric circles were drawn around the microglia at increments of $20 \mu \mathrm{m}$. The puncta number within each concentric circle were counted by Sholl analysis. Synaptic density was calculated by puncta number/neurites length in the given area. All images were processes using ImageJ. Researchers were blinded to genotype during imaging.

Plaque and plaque-associated microglia. Mice brain section was labeled with Iba-1 and $6 \mathrm{E} 10$ antibody followed by standard immunostaining procedures ${ }^{42}$. Images were captured using $10 \times$ objective and numbers of plaque were counted using ImageJ. For microglia recruitment assay, plaques were selected according to their size and divided into two groups $(20-50 \mu \mathrm{m}$ diameter group and $50-80 \mu \mathrm{m}$ diameter group). Area of plaque-associated microglia was determined by Iba- 1 staining and plaque area was calculated as $6 \mathrm{E} 10^{+}$area. Ratio of Iba- $1^{+}$area/ $6 \mathrm{E} 10^{+}$ area in the region of interest were measured by ImageJ.

Fluorescent intensity. Images were converted into 8-bit to obtain total gray value in given area by ImageJ software. Fluorescent intensity was calculated as follows: gray value (SIRPa or CD47)/(Iba-1 or NeuN)

3D Microglial Engulfment quantification. For microglial engulfment assay, z-stack images (at $0.3 \mu \mathrm{m}$ intervals) were captured by Leica TCS SP8-MaiTai MP confocal microscope with a $40 \times$ or $63 \times$ lens. Images were captured by selecting microglia with Iba-1 positive channel randomly without bias. Images were processed by smoothing and subtracting background using ImageJ software. Afterwards, 3D volume surface renderings of each z-stack were created using Imaris 7.4.2 software (Bitplane). The volume of the microglia and synaptic puncta was calculated using surface rendered images. Engulfment percentage was calculated as volume of internalized synaptic puncta/volume of microglial cell. Researchers were blinded to genotype during imaging. To examine $\mathrm{CD} 47^{+} / \mathrm{PSD} 5^{+}$puncta in microglia, double surface rendering was conducted. The first surface rendering preserved CD47 and PSD95 positive signal inside microglia. The second surface rendering was applied to check colocalization of CD47 and PSD95 signal inside the cell.

$2 D$ microglial engulfment assay in vitro. Engulfment was analyzed by calculating the fraction of the Iba- 1 area that was overlapped by the pHrodo area.

Cells were imaged using Leica TCS SP8-MaiTai MP confocal microscope. Researchers were blinded to genotype during imaging.

Human tissue samples. Human frontal lobe tissue lysate (GTX26550 for AD patients; GTX28727 and GTX15360 for controls) was purchased from GeneTex, Inc.

Table 1 Information of brain tissues from AD patients and control subjects.

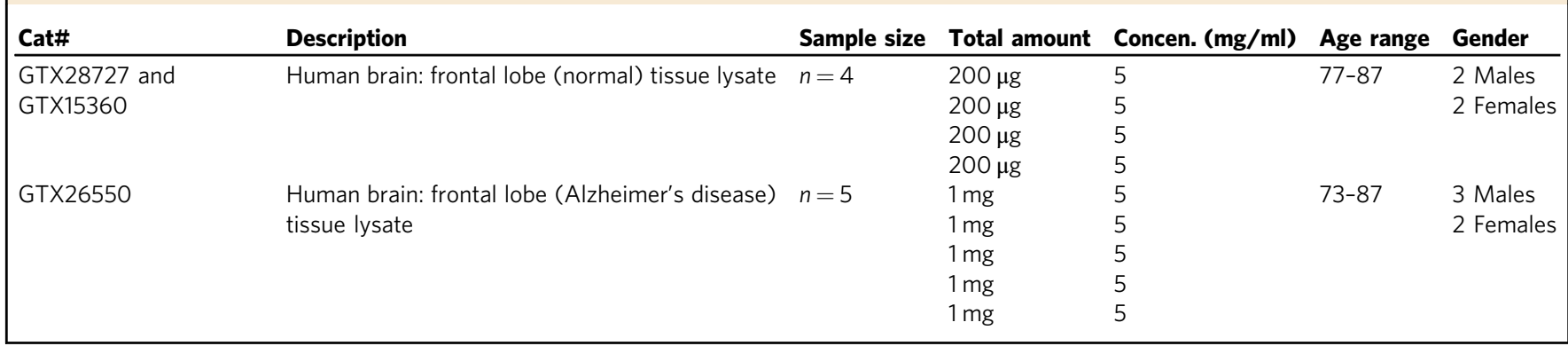


(Irvine, CA). Information about the sample is shown in Table 1 below. The homogenized lysate $(20 \mu \mathrm{g})$ was mixed with $5 \times$ protein loading buffer before boiling at $95^{\circ} \mathrm{C}$ for $10 \mathrm{~min}$ and was subjected to $10 \%$ SDS-PAGE followed by immunoblotting.

Ethics statement. The use of human tissue in this study was approved by the Ethics Review Committee of Nanjing Drum Tower Hospital Affiliated to Nanjing University Medical School.

Statistical analysis. GraphPad Prism 8 software and GPower 3.1 software were used for statistical analysis. All data were confirmed normal distribution by D'Agostino and Pearson/Shapiro-Wilk/Kolmogorov-Smirnov normality tests. Data were analyzed using one-way ANOVA (followed by Tukey's multiple comparison test or Dunnett's multiple comparisons test), two-way repeated-measures ANOVA (followed by Tukey's multiple comparisons test), two-way ANOVA (followed by Sidak's multiple comparisons test) or unpaired two-tailed $t$ test. When normality of samples failed, Kruskal-Wallis test (followed by Dunn's multiple comparisons) was performed. All $p$ values and statistical test values were indicated in Supplementary Statistical Data. All data with significant difference were analyzed by GPower to obtain effect size and power value $(>0.8)$.

Reporting summary. Further information on research design is available in the Nature Research Reporting Summary linked to this article.

\section{Data availability}

All relevant data generated for this study are included in the article/Supplementary Material/Source Data File. Other data/materials (including SIRPa fl/fl mice) that support the findings of this study are readily available from the corresponding author upon reasonable request. (Applicants should sign the material transfer agreement and take charge of all the cost regarding to mouse transfer). Source data are provided with this paper.

Received: 24 December 2019; Accepted: 9 March 2021; Published online: 01 April 2021

\section{References}

1. Kettenmann, H., Kirchhoff, F. \& Verkhratsky, A. Microglia: new roles for the synaptic stripper. Neuron $77,10-18$ (2013).

2. Paolicelli, R. C. et al. Synaptic pruning by microglia is necessary for normal brain development. Science 333, 1456-1458 (2011).

3. Schafer, D. P. et al. Microglia sculpt postnatal neural circuits in an activity and complement-dependent manner. Neuron 74, 691-705 (2012).

4. Kim, H. J. et al. Deficient autophagy in microglia impairs synaptic pruning and causes social behavioral defects. Mol. Psychiatry 22, 1576-1584 (2017).

5. Zhan, Y. et al. Deficient neuron-microglia signaling results in impaired functional brain connectivity and social behavior. Nat. Neurosci. 17, 400-406 (2014).

6. Sellgren, C. M. et al. Increased synapse elimination by microglia in schizophrenia patient-derived models of synaptic pruning. Nat. Neurosci. 22, 374-385 (2019).

7. Colonna, M. \& Butovsky, O. Microglia function in the central nervous system during health and neurodegeneration. Annu Rev. Immunol. 35, 441-468 (2017).

8. Hong, S., Dissing-Olesen, L. \& Stevens, B. New insights on the role of microglia in synaptic pruning in health and disease. Curr. Opin. Neurobiol. 36, 128-134 (2016).

9. Lehrman, E. K. et al. CD47 protects synapses from excess microglia-mediated pruning during development. Neuron 100, 120-134 e126 (2018).

10. Veerhuis, R., Nielsen, H. M. \& Tenner, A. J. Complement in the brain. Mol. Immunol. 48, 1592-1603 (2011).

11. Lui, H. et al. Progranulin deficiency promotes circuit-specific synaptic pruning by microglia via complement activation. Cell 165, 921-935 (2016).

12. Stephan, A. H., Barres, B. A. \& Stevens, B. The complement system: an unexpected role in synaptic pruning during development and disease. Annu. Rev. Neurosci. 35, 369-389 (2012).

13. Hammond, T. R., Robinton, D. \& Stevens, B. Microglia and the brain: complementary partners in development and disease. Annu Rev. Cell Dev. Biol. 34, 523-544 (2018).

14. Rogers, J. T. et al. CX3CR1 deficiency leads to impairment of hippocampal cognitive function and synaptic plasticity. J. Neurosci. 31, 16241-16250 (2011).

15. Shi, Q. et al. Complement C3 deficiency protects against neurodegeneration in aged plaque-rich APP/PS1 mice. Sci. Transl. Med. https://doi.org/10.1126/ scitranslmed.aaf6295 (2017).
16. Vasek, M. J. et al. A complement-microglial axis drives synapse loss during virus-induced memory impairment. Nature 534, 538-543 (2016).

17. Terry, R. D. et al. Physical basis of cognitive alterations in Alzheimer's disease: synapse loss is the major correlate of cognitive impairment. Ann. Neurol. 30, 572-580 (1991).

18. Hong, S. et al. Complement and microglia mediate early synapse loss in Alzheimer mouse models. Science 352, 712-716 (2016).

19. Hammond, J. W. et al. HIV Tat causes synapse loss in a mouse model of HIVassociated neurocognitive disorder that is independent of the classical complement cascade component C1q. Glia 66, 2563-2574 (2018).

20. Presumey, J. et al. Complement system in neural synapse elimination in development and disease. Adv. Immunol. 135, 53-79 (2017).

21. Barclay, A. N. \& Van den Berg, T. K. The interaction between signal regulatory protein alpha (SIRPalpha) and CD47: structure, function, and therapeutic target. Annu. Rev. Immunol. 32, 25-50 (2014).

22. Wang, J. et al. SIRPalpha deficiency accelerates the pathologic process in models of Parkinson disease. Glia 67, 2343-2359 (2019).

23. Torres, L. et al. Dynamic microglial modulation of spatial learning and social behavior. Brain Behav. Immun. 55, 6-16 (2016).

24. Wu, Y., Dissing-Olesen, L., MacVicar, B. A. \& Stevens, B. Microglia: dynamic mediators of synapse development and plasticity. Trends Immunol. 36, 605-613 (2015)

25. $\mathrm{Fu}, \mathrm{H}$. et al. Complement component $\mathrm{C} 3$ and complement receptor type 3 contribute to the phagocytosis and clearance of fibrillar Abeta by microglia. Glia 60, 993-1003 (2012).

26. Squarzoni, P. et al. Microglia modulate wiring of the embryonic forebrain. Cell Rep. 8, 1271-1279 (2014).

27. Van Hove, H. et al. Identifying the variables that drive tamoxifen-independent CreERT2 recombination: implications for microglial fate mapping and gene deletions. Eur. J. Immunol. 50, 459-463 (2020).

28. Li, Q. \& Barres, B. A. Microglia and macrophages in brain homeostasis and disease. Nat. Rev. Immunol. 18, 225-242 (2018).

29. Matcovitch-Natan, O. et al. Microglia development follows a stepwise program to regulate brain homeostasis. Science 353, aad8670 (2016).

30. Toth, A. B. et al. Synapse maturation by activity-dependent ectodomain shedding of SIRPalpha. Nat. Neurosci. 16, 1417-1425 (2013).

31. Druart, M. \& Le Magueresse, C. Emerging roles of complement in psychiatric disorders. Front. Psychiatry 10, 573 (2019).

32. Gaikwad, S. et al. Signal regulatory protein-beta1: a microglial modulator of phagocytosis in Alzheimer's disease. Am. J. Pathol. 175, 2528-2539 (2009).

33. Zhang, H., Li, F., Yang, Y., Chen, J. \& Hu, X. SIRP/CD47 signaling in neurological disorders. Brain Res. 1623, 74-80 (2015)

34. Selkoe, D. J. Alzheimer's disease is a synaptic failure. Science 298, 789-791 (2002).

35. Liu, Y. U. et al. Neuronal network activity controls microglial process surveillance in awake mice via norepinephrine signaling. Nat. Neurosci. 22, 1771-1781 (2019).

36. Stowell, R. D. et al. Noradrenergic signaling in the wakeful state inhibits microglial surveillance and synaptic plasticity in the mouse visual cortex. Nat. Neurosci. 22, 1782-1792 (2019).

37. Bian, Z. et al. Cd47-Sirpalpha interaction and IL-10 constrain inflammationinduced macrophage phagocytosis of healthy self-cells. Proc. Natl Acad. Sci. USA 113, E5434-E5443 (2016).

38. Feng, M. et al. Phagocytosis checkpoints as new targets for cancer immunotherapy. Nat. Rev. Cancer 19, 568-586 (2019).

39. Pitulescu, M. E., Schmidt, I., Benedito, R. \& Adams, R. H. Inducible gene targeting in the neonatal vasculature and analysis of retinal angiogenesis in mice. Nat. Protoc. 5, 1518-1534 (2010).

40. Stevens, B. et al. The classical complement cascade mediates CNS synapse elimination. Cell 131, 1164-1178 (2007)

41. Zhao, Y. et al. TREM2 is a receptor for beta-amyloid that mediates microglial function. Neuron 97, 1023-1031.e1027 (2018).

42. Spangenberg, E. E. et al. Eliminating microglia in Alzheimer's mice prevents neuronal loss without modulating amyloid-beta pathology. Brain 139, $1265-1281$ (2016)

\section{Acknowledgements}

This work was supported by grants from the Ministry of Science and Technology of China (2018YFA0507100), National Science Foundation of China (31741053 and 81370926), and China Postdoctoral Science Foundation funded project (2020M671449).

\section{Author contributions}

L.L. and K.Z. conceived and designed the experiments. X.D., J.W., M.H. and Z.C. performed the experiments. X.D., J.L. and Q.P.Z. analyzed the data. L.L., C.Y.Z. and Y.X. contributed reagents/materials/analysis tools. K.Z. and L.L. wrote and edited the paper.

\section{Competing interests}

The authors declare no competing interests. 


\section{Additional information}

Supplementary information The online version contains supplementary material available at https://doi.org/10.1038/s41467-021-22301-1.

Correspondence and requests for materials should be addressed to K.Z. or L.L.

Peer review information Nature Communications thanks Andrea Tenner, Hisashi Umemori and Michael Carroll for their contribution to the peer review of this work. Peer reviewer reports are available.

Reprints and permission information is available at http://www.nature.com/reprints

Publisher's note Springer Nature remains neutral with regard to jurisdictional claims in published maps and institutional affiliations. (c) (1) Open Access This article is licensed under a Creative Commons Attribution 4.0 International License, which permits use, sharing, adaptation, distribution and reproduction in any medium or format, as long as you give appropriate credit to the original author(s) and the source, provide a link to the Creative Commons license, and indicate if changes were made. The images or other third party material in this article are included in the article's Creative Commons license, unless indicated otherwise in a credit line to the material. If material is not included in the article's Creative Commons license and your intended use is not permitted by statutory regulation or exceeds the permitted use, you will need to obtain permission directly from the copyright holder. To view a copy of this license, visit http://creativecommons.org/ licenses/by/4.0/.

(C) The Author(s) 2021 